\title{
Selective Short-Term Memory Deficits Arise From Impaired Domain-General Semantic Control Mechanisms
}

\author{
Paul Hoffman \\ University of Manchester
}

\author{
Elizabeth Jefferies \\ University of York
}

\author{
Sheeba Ehsan, Samantha Hopper, and Matthew A. Lambon Ralph \\ University of Manchester
}

\begin{abstract}
Semantic short-term memory (STM) patients have a reduced ability to retain semantic information over brief delays but perform well on other semantic tasks; this pattern suggests damage to a dedicated buffer for semantic information. Alternatively, these difficulties may arise from mild disruption to domaingeneral semantic processes that have their greatest impact on demanding STM tasks. In this study, mild semantic processing impairments were demonstrated in 2 semantic STM patients. They performed well on untimed semantic tasks but were deficient in accuracy and reaction times on speeded tasks. Demanding semantic production tasks were also affected. These patients were compared with a case series of individuals with semantic aphasia whose multimodal semantic difficulties stemmed from poor cognitive control. STM and semantic performance were more impaired in this group, but there were qualitative similarities to the semantic STM patients. The difference between the 2 patient types may be a matter of degree. In semantic aphasia, severe disruption to semantic control leads to global semantic impairments, whereas in semantic STM milder disruption might impact mainly on STM tests because of the high control demands of these tasks.
\end{abstract}

Keywords: short-term memory, stroke aphasia, semantic control

Models of verbal short-term memory (STM) have traditionally emphasized the importance of phonology in list recall (e.g., Baddeley \& Hitch, 1974), but more recent research has established a role for lexical and semantic knowledge in repetition tasks (Bourassa \& Besner, 1994; Hulme, Maughan, \& Brown, 1991; Hulme et al., 1997; Jefferies, Frankish, \& Lambon Ralph, 2006; Poirier \& Saint-Aubin, 1995; Walker \& Hulme, 1999). Neuropsychological studies have a bearing on this issue, as semantic memory impairments in semantic dementia (SD) and stroke aphasia are associated with particular STM deficits (Berthier, 2001; Jefferies, Hoffman, Jones, \& Lambon Ralph, 2008; Jefferies, Jones, Bateman, \& Lambon Ralph, 2004; Knott, Patterson, \& Hodges, 1997, 2000; Majerus, Norris, \& Patterson, 2007; N. Martin \& Ayala, 2004; N. Martin \& Saffran, 1990, 1997; McCarthy \& Warrington, 1987, 2001; Patterson, Graham, \& Hodges, 1994).

Paul Hoffman, Sheeba Ehsan, Samantha Hopper, and Matthew A. Lambon Ralph, Neuroscience and Aphasia Research Unit, University of Manchester, Manchester, United Kingdom; Elizabeth Jefferies, Department of Psychology, University of York, York, United Kingdom.

The research was supported by Medical Research Council Grant G0501632 and National Institute of Mental Health Grant MH64445. We are indebted to the patients and their carers for their generous assistance with this study. We thank Linda Collier and Claire Slinger for referring some of the patients to us and Richard Wise for his assistance in interpreting brain scans.

Correspondence concerning this article should be addressed to Paul Hoffman, School of Psychological Sciences, University of Manchester, Oxford Road, Manchester M13 9PL, United Kingdom. E-mail: paul.hoffman@postgrad.manchester.ac.uk
A key feature in both of these groups is a reduction or abolition of the normal effect of "lexicality" - the advantage healthy individuals receive when repeating word lists relative to lists of nonwords as a result of their lexical-semantic knowledge (Hulme et al., 1991). This reduction is in line with the idea that lexicalsemantic contributions to STM are disrupted in patients with impaired semantic memory (Patterson et al., 1994). Intriguingly, there are a handful of stroke patients reported who have shown a reduced lexicality effect and other difficulties in retaining semantic information over time but who have performed well on other semantic tasks (Freedman \& Martin, 2001; R. C. Martin \& He, 2004; R. C. Martin \& Lesch, 1996; R. C. Martin, Shelton, \& Yaffee, 1994). This pattern has been termed a "semantic STM deficit," because the lexical-semantic aspects of STM are selectively impaired but long-term semantic knowledge appears relatively preserved. The nature of this unusual deficit and its relation to other semantic disorders are our focus in this study.

R. C. Martin et al. (1994) first reported the case of an individual with a semantic STM deficit. Patient AB showed no lexicality effect, and this indicated a problem with lexical-semantic aspects of STM. In contrast, his recall was sensitive to word length and phonological similarity, which are both indicators of normal phonological retention (Baddeley, Thompson, \& Buchanan, 1975; Conrad, 1964; Conrad \& Hull, 1964). AB scored within the normal range on tests of picture naming and single-word comprehension, and this fact suggested that semantic processing more generally was spared. The performance of this patient was contrasted with that of EA, who had deficits in phonological retention. She showed no effects of word length or phonological similarity in her imme- 
diate serial recall and recalled lists of nonwords much more poorly than lists of real words.

The differences between the two patients were exemplified by their performance on two tasks that explicitly tapped either phonological or semantic storage. In a rhyme probe task, patients heard a list of words and verified whether a probe rhymed with any of them. In a category probe task, patients heard a list of words and verified whether a subsequent probe belonged to the same semantic category as any of them. The two tasks therefore emphasized retention of either the phonological or semantic characteristics of the list items, respectively. Although the patients showed abnormal performance on both tasks, $\mathrm{AB}$ was more impaired on the category than the rhyme task and EA showed the reverse dissociation. Later, an additional patient, ML, was reported who presented a similar semantic STM deficit to that of AB (R. C. Martin \& He, 2004; R. C. Martin \& Lesch, 1996). ML's ability to name pictures and comprehend single words was also good.

Martin and colleagues attributed the deficit in $\mathrm{AB}$ and ML to the disruption of a STM store specialized for the retention of lexicalsemantic information (R. C. Martin, Lesch, \& Bartha, 1999; R. C. Martin et al., 1994). This multicomponent model of STM explains the dissociation between lexical-semantic STM impairment and phonological STM impairment, which forms the major focus of existing research on semantic STM cases. By positing a separate system for short-term retention of semantic information that is independent of the mechanisms underlying performance on other semantic tasks, the account also fits the apparently selective deficits to STM in these patients. However, an alternative perspective, and one that has not been previously been explored in detail, is that semantic STM deficits are a consequence of disruption to more general semantic processes. On this view, semantic STM patients do have general deficits of semantic processing, albeit rather subtle ones that may not be detected on standard semantic tasks. The impact of this mild semantic impairment is observed on semantic STM tasks and not on tests of phonological STM (or on most single-word semantic tasks), simply because semantic STM tasks place the greatest demands on the semantic system. On this view, impairment would be expected on other semantic tasks that were sufficiently demanding, irrespective of their STM load.

There are some hints that semantic STM patients have difficulty with demanding tasks that do not load heavily on STM. Patient AB was error prone when verifying whether a word belonged to a particular semantic category but only when the stimuli were presented for a limited time period (R. C. Martin et al., 1994). His reaction times were slower than those of controls on this and other speeded comprehension tests (living/nonliving judgments and verification of property statements such as "belt has buckle"). Patient ML showed slow response times on the category verification task (R. C. Martin \& He, 2004). In the present study, we explored in two new semantic STM cases the hypothesis that the semantic STM profile is the consequence of a mild general semantic impairment. Rather than focus on the dissociation between semantic and phonological STM impairments, we compared our semantic STM patients with a case series of patients with stroke aphasia who had multimodal semantic deficits to determine to what extent a similar underlying impairment could account for both sets of symptoms. To put our investigation into context, we must address at the outset the following key question: Why would we expect a mild semantic impairment to affect semantic STM tasks to a greater extent than other semantic tasks? To do so, we must depart from the domain of STM to consider how the semantic system itself is organized.

\section{Disorders of Semantic Representation Versus Semantic Control}

Disorders of comprehension are present in a number of conditions, and their precise form can be rather variable. Some patients have modality-specific comprehension impairments (e.g., pure word deafness) that can be attributed to presemantic levels of processing. In other cases, semantic processing is impaired across a range of input and output modalities, and this pattern of impairment implicates damage to a central semantic system. These multimodal semantic impairments are most commonly associated with $\mathrm{SD}$, a neurodegenerative condition in which core semantic knowledge degrades progressively in a very selective manner (Hodges, Patterson, Oxbury, \& Funnell, 1992; Rogers et al., 2004; Snowden, Goulding, \& Neary, 1989). Comprehension impairments affecting both verbal and nonverbal modalities can also occur in stroke aphasia (Chertkow, Bub, Deaudon, \& Whitehead, 1997; De Renzi, Faglioni, Scotti, \& Spinnler, 1972; Gainotti, Miceli, \& Caltagirone, 1979; Gainotti, Silveri, Villa, \& Caltagirone, 1983; Hart \& Gordon, 1990; Saygin, Dick, Wilson, Dronkers, \& Bates, 2003; Spinnler \& Vignolo, 1966), and, as a convenient shorthand, we will refer to the impairment in these cases as semantic aphasia (SA). ${ }^{1}$ Despite the apparent symptom overlap between SD and SA, distinct brain regions are involved in each condition. In SD, atrophy and hypometabolism are centered bilaterally on the anterior temporal lobes (Mummery et al., 2000; Nestor, Fryer, \& Hodges, 2006). In contrast, semantic impairments in stroke aphasia have been associated with frontal and temporoparietal infarcts (e.g., Berthier, 2001; Chertkow et al., 1997).

In a recent study, a case-series methodology was used to compare semantic performance in SD and SA directly (Jefferies \& Lambon Ralph, 2006). Although the two groups showed similar overall levels of impairment, qualitative differences emerged. The SD patients showed substantial consistency when the same set of items was assessed across different semantic tests. They were also highly sensitive to frequency in a range of tasks. These findings are consistent with the view that SD patients suffer degradation of a store of amodal semantic representations (Patterson, Nestor, \& Rogers, 2007; Rogers et al., 2004). The SA patients, in contrast, did not perform consistently on tasks that required different types of semantic judgment (e.g., simple matching tasks vs. judgments of semantic association) even when the same concepts were probed, and this result suggests that their deficits did not stem from loss of the knowledge store itself. They often succeeded in retrieving the semantic information required for one task but then were unable to reshape the information to meet the requirements of another task. In contrast to the SD patients, they were not sensitive to item frequency.

Further investigations have revealed that, unlike SD patients, SA patients show "refractory" effects in semantic tasks (i.e., their performance deteriorates when a small set of semantically related

${ }^{1}$ This term is not intended to exclude the possibility that individual patients of this sort may have additional nonsemantic deficits. 
items is presented several times; Jefferies, Baker, Doran, \& Lambon Ralph, 2007; see also Warrington \& Cipolotti, 1996). These characteristics have been associated with impaired access to semantic information rather than with damage to the semantic store itself (Warrington \& McCarthy, 1983; Warrington \& Shallice, 1979).

These contrasting patterns of performance were consistent with the hypothesis that semantic cognition consists of two interactive components: (a) amodal semantic representations that code our knowledge of concepts and their interrelationships and (b) control processes that access and manipulate these representations to achieve appropriate semantically driven behavior. In SD, semantic representations in the anterior temporal lobes degrade and are no longer available to drive semantic performance. Because the same representations are required irrespective of the precise nature of the task, this degradation leads to a high level of consistency between different tasks. However, intact semantic representations alone cannot drive appropriate semantic behavior. We know many things about objects, and only a small subset of this vast store of information is relevant at any given time. To borrow an example from Saffran (2000), if we are asked to play a piano, it is necessary for us to access information about the functions of the keys and the pedals. But if our task is to move the piano across the room, a different set of piano-related features becomes relevant: It is now important to know that pianos are heavy and that they usually move on wheels.

Our view is that semantic control processes allow us to access and manipulate semantic knowledge in a flexible and taskappropriate manner. Thus, they ensure that the relevant aspects of knowledge are processed and that irrelevant ones are ignored. An impairment of this function can explain the pattern of deficits seen in SA (Jefferies \& Lambon Ralph, 2006). SA patients' inconsistency across different kinds of semantic judgment would be expected if different tasks placed varying demands on control processes. The effects of varying control demands were apparent on a semantic association task (the Camels and Cactus Test; Bozeat, Lambon Ralph, Patterson, Garrard, \& Hodges, 2000): SA patients (but not SD patients) performed most poorly on those trials in which it was difficult to elucidate the relevant semantic relationship and reject the distractors.

The errors made by SD patients and SA patients in picture naming were consistent with the distinction between degraded knowledge and impaired control. Both groups produced predominately semantic rather than phonological errors, and this indicated a semantic locus of impairment. However, whereas SD patients made coordinate errors (cow $\rightarrow$ "horse") and superordinate errors (cow $\rightarrow$ "animal"), SA patients made additional errors of an associative nature (e.g., squirrel $\rightarrow$ "nuts," glass $\rightarrow$ "ice," lorry $\rightarrow$ "diesel") that almost never occurred in SD patients.

Patients with SD typically incorrectly named objects as highly familiar and prototypical members of their class (e.g., naming all small mammals as "dog" or "cat"; Hodges, Graham, \& Patterson, 1995; Woollams, Cooper-Pye, Hodges, \& Patterson, 2008). This behavior reflects the fact that fine-grained, item-specific knowledge degrades early on in this condition; therefore, the damaged semantic system is likely to be driven by highly typical features that are common to many exemplars (Rogers et al., 2004). Associative errors occur very rarely, as they are indicative of objectspecific knowledge that is lost in SD (e.g., to produce "nuts" in response to a picture of a squirrel, the semantic system must contain some fairly specific information about the feeding habits of squirrels).

In contrast, SA patients did produce these sorts of responses, and this fact suggests that they continued to activate some itemspecific information and, therefore, that the underlying store of semantic knowledge was relatively intact. However, SA patients appeared to have difficulty regulating their semantic activation in order to select the appropriate target name over other highly active but task-irrelevant responses. The naming by SA patients improved substantially when they were provided with phonological cues, and this fact again indicated that knowledge about the target names was still present within the semantic system. These cues may have acted as a source of external constraint over activation in the semantic system that reduced the internal control requirements necessary for selecting the correct response (Jefferies \& Lambon Ralph, 2006; Jefferies, Patterson, \& Lambon Ralph, 2008). Cuing produced much smaller effects in patients with SD, presumably because little phonological information was generated from semantics to combine with the phonemic cues.

This semantic control impairment aligns with a large body of neuroimaging evidence implicating the left inferior prefrontal cortex in controlled semantic processing (for reviews, see Badre \& Wagner, 2002; Thompson-Schill, Bedny, \& Goldberg, 2005). This region is commonly activated in semantic tasks and shows increased activation when semantic tasks require selection among competing alternatives (Moss et al., 2005; Thompson-Schill, D'Esposito, Aguirre, \& Farah, 1997; Thompson-Schill, D'Esposito, \& Kan, 1999) or semantic retrieval from weak or poorly specified cues (Bunge, Wendelken, Badre, \& Wagner, 2005; Wagner, Pare-Blagoev, Clark, \& Poldrack, 2001). Left prefrontal lesions were present in the majority of SA patients studied by Jefferies and Lambon Ralph (2006), but 2 patients presented with damage only in posterior areas and centered on the temporoparietal junction (most of the other patients had large lesions encroaching on both areas). The consequences of these diverse lesions on semantic cognition were very similar: Patients showed poor semantic control regardless of whether prefrontal or temporoparietal cortex was damaged (see also Berthier, 2001). This finding suggests that semantic control may involve a distributed network of brain regions. This proposal is consistent with recent approaches to cognitive and executive control more generally.

Although cognitive control has traditionally been associated exclusively with the prefrontal cortices, recent work has highlighted the importance of a distributed network of frontal and temporoparietal regions (Collette, Hogge, Salmon, \& Van der Linden, 2006; Peers et al., 2005). Prefrontal and temporoparietal cortex show coupled activation during the performance of executive tasks, particularly in the context of working memory (Collette et al., 2005; Collette \& Van der Linden, 2002; Crosson et al., 1999; Garavan, Ross, Li, \& Stein, 2000).

\section{Relevance to STM Deficits}

These findings show that damage to core semantic representations (in SD) dissociates from impairment to the control processes that are recruited to access and manipulate these representations (in SA). Could either of these impairments explain the semantic STM profile in which semantic performance is disproportionately 
affected when multiple items must be retained over a delay? The degradation of semantic representations that lies at the heart of SD is an unlikely candidate, because STM impairments in this condition are closely related to the status of individual word meanings. Although SD is associated with poor immediate serial recall (ISR) for words, this deficit is restricted to poorly understood words that are also failed in comprehension tasks (Jefferies et al., 2004; Patterson et al., 1994). This situation is rather different from that in semantic STM patients, who show poor recall of words even when comprehension of individual word meanings is intact. Furthermore, the errors made by patients with SD are primarily phoneme migrations in which segments of phonology from different words are blended together (Jefferies, Hoffman, et al., 2008; Knott et al., 1997; Majerus et al., 2007; Patterson et al., 1994). Semantic STM patients, on the other hand, usually make omissions, serial order errors, or intrusions from previous lists in ISR (R. C. Martin \& Lesch, 1996).

The alternative semantic deficit, impairment to control processes observed in SA cases, is more promising. SA patients are inconsistent across tests with different task demands, even when the same words are being probed, because their success depends on how much control is required to access and manipulate the semantic representations required for the task in hand (Jefferies \& Lambon Ralph, 2006). Verbal STM tasks, in which multiple words must be activated and maintained simultaneously, might be expected to place greater demands on control processes than do standard semantic tasks that feature single words. Indeed, when we tested a subset of the SA patients described above on an ISR task, they made an abnormally high number of serial order errors (Jefferies, Hoffman, et al., 2008). Some of these patients consistently distorted the list order in a particular way by beginning their response with the final list item. This pattern might reflect an inability to resolve competition between different activated list items, in line with proposals that cognitive control mechanisms support serial order memory when span is exceeded (Hazy, Frank, \& O'Reilly, 2006; O'Reilly, Braver, \& Cohen, 1999; O'Reilly \& Soto, 2002). Rather than direct their attention first to early list items, patients immediately responded with the final list item, which presumably was most accessible as a result of its recent presentation.

A similar explanation has been proposed for the same error pattern in semantic STM patient AB: R. C. Martin and Lesch (1996) suggested that $A B$ suffered from a failure of inhibition. More recent work has revealed poor inhibition in semantic STM patient ML (Hamilton \& Martin, 2005, 2007). ML is highly susceptible to proactive interference in STM tasks, and this susceptibility reflects an inability to inhibit strongly activated but irrelevant representations and suggests that an underlying cognitive control deficit underlies his STM problems. Our hypothesis in this study was rather similar: that semantic STM patients suffer from poor control over their semantic representations and that, as a result, their ability to maintain multiple words in STM is impaired. However, our working hypothesis was that these problems are not limited to STM and could be observed in any semantic task that placed sufficient demands on control mechanisms.

To investigate this possibility, we explored semantic processing in two individuals with semantic STM. We compared these patients with a case series of individuals with SA who exhibited poor semantic control across a range of non-STM tasks (Jefferies \&
Lambon Ralph, 2006). We expected the semantic STM patients to show mild semantic difficulties on tasks, irrespective of the STM load, and we expected these difficulties to be most pronounced on tasks that required a high degree of semantic control (e.g., retrieval from a weakly specified cue or selection from highly activated competitors). The individuals with SA were tested with the same tasks to establish whether their pattern of impairment was similar.

There were two components to our investigation. First, we investigated verbal STM in the two semantic STM patients to establish that their deficits related to poor recall of lexicalsemantic information. The patients with SA completed the same tasks, and we expected that they would show a similar pattern of impairment as a result of their semantic control deficits. Second, we examined semantic processing using a range of tasks. We began by using a standard test battery that has previously revealed multimodal semantic deficits in SA and SD (Bozeat et al., 2000; Jefferies \& Lambon Ralph, 2006). Semantic performance was also tested under more demanding conditions with further tests that emphasized speeded responding. In these tests, we minimized the involvement of STM by using visual presentation, as this allowed us to assess whether semantic STM patients have a more general difficulty in completing semantic tasks under demanding conditions. Finally, we gave the patients word production tasks in which cognitive control was required for successful task completion. We expected that performance in the semantic STM cases and the SA group would worsen as control requirements increased.

\section{Case Descriptions}

A summary of biographical and neuroimaging information and aphasia classifications for all patients is given in Table 1 .

\section{Semantic STM Patient JB}

JB was a 51-year-old man who left school at age 15 and was employed as a factory foreman until he suffered a left-hemisphere hemorrhagic cerebrovascular accident (CVA) in April 2005. He was initially densely amnesic and did not recognize family members for 2 weeks post CVA. JB's language profile assessed 4 months post stroke was closest to transcortical sensory aphasia. His speech was fluent and his ability to repeat verbal material was excellent, but he displayed marked comprehension and wordfinding difficulties. He correctly named 7 of the first 17 items on the Boston Naming Test (BNT; Kaplan, Goodglass, \& Weintraub, 1983) without cues. We began the investigation reported here in February 2006, by which time his language skills had improved substantially. His comprehension and spontaneous speech appeared normal, though he still complained of occasional wordfinding difficulty and amnesia for recent events. At that point, he named 42/60 items on the BNT without cues.

A series of axial and coronal MRI slices for JB is shown in Figure 1 (radiological convention with the left hemisphere shown on the right). The damage resulting from the hemorrhage was confined to the left hemisphere. The superior temporal gyrus and sulcus were intact along their lengths, and the anterior part of the temporal lobe was also intact. Loss of tissue was primarily along the fusiform and inferior temporal gyri (including underlying white matter) and, to a lesser extent, the middle temporal gyrus. The parahippocampal, perirhinal, and entorhinal cortices seemed 
Table 1

Background Details

\begin{tabular}{|c|c|c|c|c|c|c|}
\hline Group and patient & Gender & Age (years) & Education (leaving age) & Neuroimaging summary & Years since CVA & Aphasia type \\
\hline \multicolumn{7}{|l|}{ Semantic STM } \\
\hline JB & M & 51 & 15 & Left temporal & 2 & NA \\
\hline $\mathrm{ABU}$ & $\mathrm{M}$ & 52 & 15 & Nonspecific & 4 & NA \\
\hline \multicolumn{7}{|l|}{ SA } \\
\hline JD & M & 80 & 18 & NA & 9 & NA \\
\hline NY & M & 63 & 15 & L frontal-temporal-parietal & 4.5 & Conduction \\
\hline $\mathrm{SC}$ & M & 76 & 16 & $\begin{array}{l}\text { Left occipital-temporal (and right } \\
\text { frontal-parietal) }\end{array}$ & 5.5 & Anomic/TSA \\
\hline PG & M & 59 & 18 & Left frontal and capsular & 5 & TSA \\
\hline $\mathrm{KH}$ & M & 73 & 14 & $\begin{array}{l}\text { Left occipital-temporal and } \\
\text { frontal }\end{array}$ & 1.5 & Mixed TA \\
\hline BB & $\mathrm{F}$ & 55 & 16 & Left frontal and capsular & 2.5 & Mixed TA \\
\hline $\mathrm{JM}$ & $\mathrm{F}$ & 69 & 18 & Left frontal-temporal-parietal & 6 & TSA \\
\hline ME & $\mathrm{F}$ & 36 & 16 & Left occipital-temporal & 6.5 & TSA \\
\hline MS & $\mathrm{F}$ & 73 & 14 & NA & 5 & Global \\
\hline LS & M & 71 & 15 & Left temporal-parietal-frontal & 3 & TSA \\
\hline KA & M & 74 & 14 & Left frontal-temporal-parietal & 1 & Global \\
\hline
\end{tabular}

Note. Patients are arranged in order of word-picture matching score (see Table 4). Aphasia classifications were determined with the Boston Diagnostic Aphasia Examination (Goodglass, 1983) and were confirmed by an experienced speech and language therapist. CVA = cerebrovascular accident; STM = short-term memory; $\mathrm{SA}=$ semantic aphasia; $\mathrm{NA}=$ not available; TSA $=$ transcortical sensory aphasia; $\mathrm{TA}=$ transcortical aphasia

to be intact, as did the hippocampus. There was also a widening of the left sylvian fissure and the posterior horn of the lateral ventricle, which may indicate some additional damage in the surrounding cortex.

\section{Semantic STM Patient ABU}

ABU was a 52-year-old man who left school at age 15 and was employed as a sheet metal worker for a number of years. He experienced a CVA in June 2003 that resulted in right-sided hemiparesis. A computerized tomography scan obtained at this time was inconclusive. The left lateral ventricle appeared enlarged and the gray-white matter contrast in the basal ganglia was reduced on the left side, which could indicate a diffuse lefthemisphere partial infarction. We were unable to obtain an MRI scan, due to counterindications. ABU presented acutely with wordfinding difficulty and mild comprehension problems. He correctly named 9 of the first 23 items in the BNT (without cues); however,

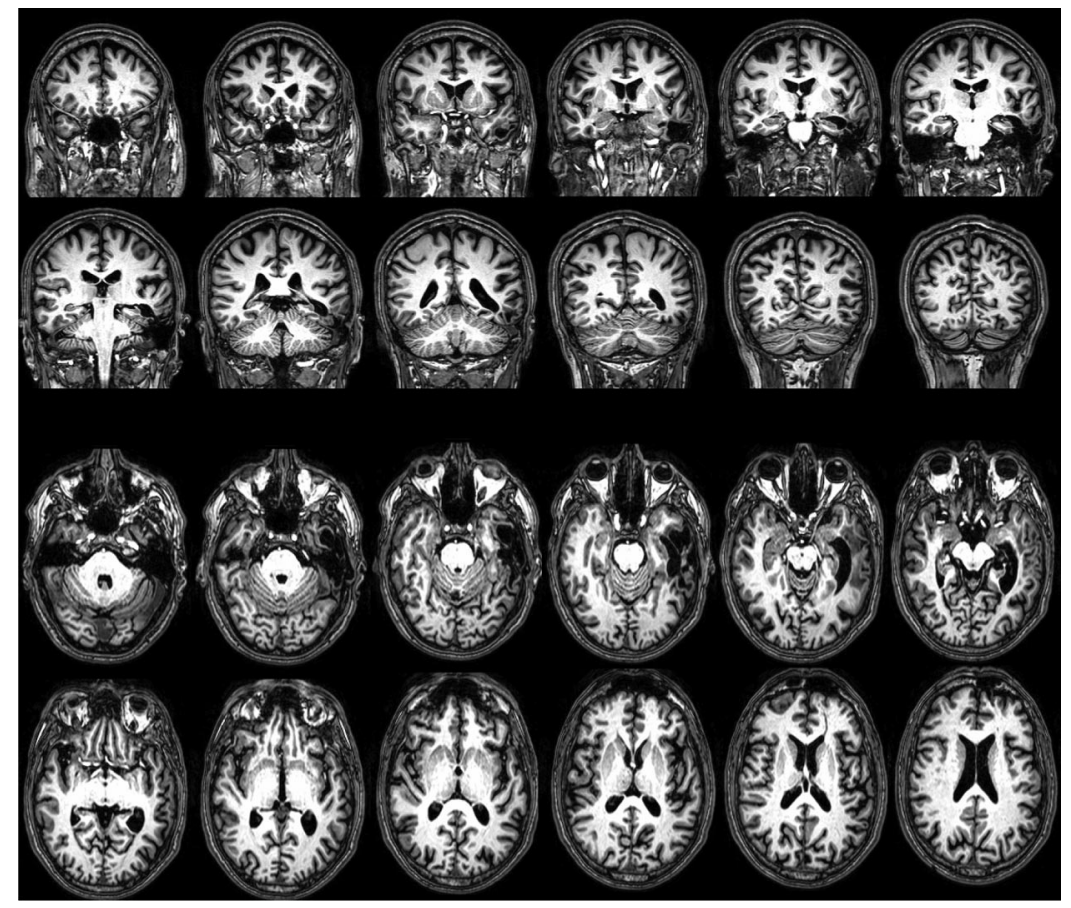

Figure 1. MRI scan for patient JB. 
his language abilities recovered after this initial period. When ABU was first seen by us in December 2006, his comprehension was good and his speech was fluent, though punctuated by occasional hesitations. His phrase length also appeared slightly reduced. He could successfully name 43/60 pictures in the BNT.

\section{SA Group: Aphasia With Multimodal Semantic Impairments}

$\mathrm{JB}$ and $\mathrm{ABU}$ were compared with a case series of 11 individuals with stroke aphasia recruited from stroke clubs and speech and language therapy services in Manchester, United Kingdom. Patients with verbal comprehension deficits were initially screened and were enrolled in the study if they failed both picture and word tests of semantic association (Camels and Cactus test; Bozeat et al., 2000). Every patient had chronic language impairment from a CVA at least a year previously. Their semantic deficits are described in more detail later in this article. Ten of these individuals have been studied previously, and their semantic problems have been shown to be associated with a failure to control semantic activation in a task-appropriate fashion (Jefferies \& Lambon Ralph, 2006). An additional case, JD, was referred to us more recently and was included in the case series for the present study. Although he failed a number of semantic tasks in multiple modalities, he was one of the least impaired patients in the case series.

\section{Control Participants}

A single set of 10 individuals served as controls for most of the experimental tasks reported in this paper. This control group had a mean age of 61 , which did not differ significantly from the mean age of the patients, $t(21)=0.70, p=.49$.

\section{Statistical Analyses}

When comparing the performance of individual patients with that of our control group, we used the modified $t$-test method recommended by Crawford and Garthwaite (2002). We also employed a number of standardized tests with existing norms. For these we used published cutoffs to determine whether a patient was impaired. If such cutoffs were not available, we considered any score more than two standard deviations below the published mean to be impaired.

\section{Background Neuropsychology}

Method. Patients were tested on a number of nonverbal attentional/executive tasks, namely Raven's Coloured Progressive Matrices (Raven, 1962), the Brixton Test of Spatial Awareness (Burgess \& Shallice, 1996), the Elevator Counting subsets of the Test of Everyday Attention (Robertson, Ward, Ridgeway, \& NimmoSmith, 1994), and the Wisconsin Card-Sorting Test (WCST; Milner, 1964; Stuss et al., 2000). These tests recruit a number of abilities associated with executive function. In the Brixton test, the patient attempts to predict the path of a moving dot through a spatial array. At various points in the test and unknown to the patient, the rules that govern the dot's movement change, requiring the patient to inhibit the previous rule and respond to the new rule. Similar processes are required in the WCST, in which sorting rules must be updated on the basis of feedback from the experimenter.
The Elevator Counting test features two conditions. The first is counting without distraction, in which the patient simply counts a series of tones. In the "with distraction" condition, high- and low-pitched tones are interspersed but the patient counts only the low-pitched tones. This task involves shifting attention between the tones and the tally and inhibiting the tendency to respond to all of the tones. Finally, Raven's Coloured Matrices is a measure of fluid intelligence in which the patient selects the appropriate block to match with a visual pattern. On the more difficult trials, the patterns vary on a number of different dimensions that the patient must attend to simultaneously and integrate in an abstract way in order to select the correct response; thus, the test is executively demanding.

Patients also received tests of forward and backward digit span (Wechsler, 1987). Their visual processing was assessed with several subtests from the Visual Object and Space Perception battery (Warrington \& James, 1991) and immediate copies of the Rey complex figure (Rey, 1941).

Results and discussion. Scores are shown in Table 2. In all tables and figures, data for semantic STM patients JB and ABU are presented on the left. Data for the semantic aphasic patients are presented on the right and are arranged in order of the patients' comprehension impairment (word-picture matching scores). There was some evidence of mild executive dysfunction in JB and ABU. JB exhibited weakness on the Raven's test and the Brixton test, whereas ABU performed poorly on the WCST, scored just above the minimum cutoff score on the Brixton test, and showed some weakness on the Elevator Counting test. As no norms existed for the age group of JB and ABU for Raven's Coloured Matrices, we also tested them with the standard matrices set (Raven, 1992). JB scored 23/60, which fell below the 5 th percentile for his age group. ABU scored 34/60, which reached the 10th percentile.

The SA patients all showed evidence of executive dysfunction, albeit in a somewhat variable manner, as different patients failed different tests. This might be because different tasks required different executive functions. Evidence from healthy individuals suggests that "executive" tasks in fact tap a number of correlated but separable functions (e.g., Miyake et al., 2000, identified separate shifting, updating, and inhibition components). Variation in patient scores might reflect variation in the degree of impairment to each of these components. Another possibility is that the patients were affected in different ways by the modalities tapped by each test (e.g., Elevator Counting uses auditory presentation and a verbal response, whereas the Brixton test is visuospatial and uses a pointing response). In line with this possibility, digit spans and visuospatial processing were impaired rather variably in the SA group. Such variance potentially reflects additional visual or phonological deficits in some patients, due to their large lesions. Poor comprehension of task instructions could have contributed to the impaired executive scores of the SA patients, although this possibility is unlikely to account for the deficits of JB and ABU. These patients have very good verbal comprehension and successfully followed complex instructions on other tasks we have presented to them. Moreover, digit spans were normal for these patients, which suggests preservation of phonological STM. JB and ABU scored well on the Visual Object and Space Perception tests, and this indicated good visuospatial skills (although ABU's copy of the Rey figure was mildly impaired). 
Table 2

Background Neuropsychology

\begin{tabular}{|c|c|c|c|c|c|c|c|c|c|c|c|c|c|c|c|c|}
\hline \multirow[b]{2}{*}{ Task } & \multirow[b]{2}{*}{ Max } & \multirow{2}{*}{$\begin{array}{l}\text { Normal } \\
\text { cutoff }\end{array}$} & \multicolumn{2}{|c|}{$\begin{array}{l}\text { Semantic } \\
\text { STM }\end{array}$} & \multicolumn{12}{|c|}{ SA } \\
\hline & & & JB & $\mathrm{ABU}$ & $M$ & JD & NY & $\mathrm{SC}$ & PG & $\mathrm{KH}$ & $\mathrm{BB}$ & JM & ME & MS & LS & KA \\
\hline \multicolumn{17}{|l|}{ Attentional/executive } \\
\hline \multicolumn{17}{|l|}{ Raven's Progressive } \\
\hline Matrices $^{\mathrm{a}}$ & & & $<5^{*}$ & 10 & & 95 & 50 & 50 & 50 & $5^{*}$ & 50 & $5^{*}$ & $<5^{*}$ & $5^{*}$ & 10 & $5^{*}$ \\
\hline $\begin{array}{l}\text { WCST (number of } \\
\text { categories) }\end{array}$ & 6 & & 6 & $3^{\dagger}$ & 1.1 & $1^{*}$ & $2^{*}$ & 6 & $0^{*}$ & $0^{*}$ & $1^{*}$ & $2^{*}$ & $0^{*}$ & $0^{*}$ & $0^{*}$ & $1^{*}$ \\
\hline $\begin{array}{l}\text { Brixton Spatial Awareness } \\
\text { (correct) }^{\mathrm{b}}\end{array}$ & 54 & 28 & $28^{*}$ & 30 & 19.0 & $28^{*}$ & 34 & $25^{*}$ & $26^{*}$ & $7^{*}$ & $23^{*}$ & NT & $11^{*}$ & $16^{*}$ & $14^{*}$ & $6^{*}$ \\
\hline $\begin{array}{l}\text { TEA: counting without } \\
\text { distraction }\end{array}$ & 7 & 5 & 7 & 7 & 4.8 & 7 & $3^{*}$ & 7 & $3^{*}$ & 6 & $4^{*}$ & $3^{*}$ & 7 & NT & $3^{*}$ & NT \\
\hline $\begin{array}{l}\text { TEA: counting with } \\
\text { distraction }\end{array}$ & 10 & 3 & 10 & 5 & 2.6 & 6 & $2^{*}$ & $1^{*}$ & $0^{*}$ & $3^{*}$ & $0^{*}$ & $0^{*}$ & 9 & NT & $2^{*}$ & NT \\
\hline \multicolumn{17}{|l|}{ Digit Span ${ }^{c}$} \\
\hline Forward & & 5 & 7 & 5 & 3.8 & 5 & $3^{*}$ & 6 & 6 & $4^{*}$ & 5 & $3^{*}$ & 6 & $0^{*}$ & $4^{*}$ & $0^{*}$ \\
\hline Backward & & 3 & 4 & 4 & 2.0 & 4 & $2^{*}$ & $2^{*}$ & $2^{*}$ & $2^{*}$ & $0^{*}$ & $2^{*}$ & 3 & NT & $1^{*}$ & NT \\
\hline \multicolumn{17}{|l|}{ Visuospatial } \\
\hline Rey figure copy ${ }^{\mathrm{d}}$ & 36 & 29.0 & 33 & 29 & 25.2 & 31 & $26^{*}$ & 35 & 36 & NT & $20^{*}$ & NT & $9.5^{*}$ & NT & $19.5^{*}$ & NT \\
\hline VOSP incomplete letters & 20 & 17.6 & 20 & 19 & 12.9 & 20 & $17^{*}$ & 18 & 18 & 19 & $0^{*}$ & $11^{*}$ & $6^{*}$ & 20 & $0^{*}$ & NT \\
\hline VOSP dot counting & 10 & 9.5 & 10 & 10 & 8.0 & 10 & 10 & 10 & $5^{*}$ & 10 & 10 & $6^{*}$ & $3^{*}$ & 10 & $6^{*}$ & NT \\
\hline VOSP position & & & & & & & & & & & & & & & & \\
\hline discrimination & 20 & 18.6 & 20 & 20 & 17.5 & 20 & 20 & $17^{*}$ & 20 & 18 & 18 & 16 & $15^{*}$ & 19 & $16^{*}$ & $14^{*}$ \\
\hline VOSP number location & 10 & & 10 & 10 & 8.0 & 10 & 10 & 10 & 9 & 9 & 8 & 8 & 2 & NT & 8 & 6 \\
\hline VOSP cube analysis & 10 & 4.7 & 10 & 9 & 5.9 & 10 & 5 & 9 & 10 & $3^{*}$ & $2^{*}$ & $4^{*}$ & $4^{*}$ & 8 & $4^{*}$ & NT \\
\hline
\end{tabular}

Note. $\quad$ STM $=$ short-term memory; SA $=$ semantic aphasia; WCST $=$ Wisconsin Card-Sorting Test (Milner, 1964; Stuss et al., 2000); TEA $=$ Test of Everyday Attention (Robertson et al., 1994); VOSP = Visual Object and Space Perception battery (Warrington \& James, 1991); NT = not tested. ${ }^{*}$ Denotes impaired scores $\left(<2\right.$ standard deviations below normal mean). ${ }^{\dagger}$ Denotes below 10th percentile.

${ }^{a}$ Refers to the standard test for JB and ABU and Raven's Coloured Progressive Matrices for the remaining patients (Raven, 1962, 1992). ${ }^{\mathrm{b}}$ Burgess and Shallice (1996). ${ }^{\mathrm{c}}$ Weschler (1987). ${ }^{\mathrm{d}}$ Rey (1941).

\section{Section 1: Verbal STM}

In the first set of experiments, we investigated verbal STM in JB and $\mathrm{ABU}$ to establish that they had particular difficulty retaining lexical-semantic information. If this was the case, we would expect them to perform well on tests requiring only phonological storage but more poorly when semantic information was relevant to recall. Thus, the profile required to class them as having semantic STM was as follows: (a) better performance on a rhyme than a category probe task, as the category task explicitly taps semantic knowledge about the words maintained in STM (R. C. Martin et al., 1994); (b) good recall of letters and nonwords in ISR, as these items are maintained in phonological form; (c) impaired ISR of word lists, relative to nonword recall (i.e., a reduced lexicality effect), because lexical-semantic knowledge makes a larger contribution to the recall of words (e.g., Hulme et al., 1991; Jefferies et al., 2004); and (d) a normal phonological similarity effect in letter ISR, as this is associated with normally functioning phonological STM (Conrad \& Hull, 1964).

We gave the same tasks to members of the semantic aphasic case series to investigate whether a similar profile of STM impairment would be present in the context of more severe semantic impairment. Five SA patients completed the ISR tasks. These 5 all fit the diagnostic criteria of transcortical sensory aphasia, which is characterized by good repetition, fluent speech, and poor comprehension (Albert, Goodglass, Helms, Rubens, \& Alexander, 1981; Berthier, 2000). Therefore, they could complete list repetition tasks without deficits of phonology or speech output dominating their results. The category and rhyme probe tasks required no spoken output and were completed by a larger number of patients (although $\mathrm{JM}, \mathrm{KH}$, and MS withdrew from the study prior to this test being completed).

\section{Category and Rhyme Probed Recall}

A hallmark of impaired semantic STM is a dissociation between tests featuring category and rhyme probes (R. C. Martin et al., 1994). Semantic STM patients are unable to verify whether words in a spoken list belong to the same category as does a probe word presented after the list, even though they can demonstrate knowledge of category membership when items are encountered individually. Demonstration of this effect in JB and ABU was important to establish their status as semantic STM patients.

Materials. Category and rhyme tasks were based on those of R. C. Martin et al. (1994). The rhyme test featured list lengths from one to nine items. Each list consisted of nonrhyming words. For half of the lists, the nonrhyming words were followed by a probe that rhymed with one of the list items (e.g., list: dog, wish, hat; probe: dish). For the remaining lists, the probe was nonrhyming. There were 20 lists for each of lengths one, two, and five and 24 lists for each of the remaining list lengths. Methods for creating the category lists were the same, except that each word belonged to one of nine categories: animals, body parts, clothing, flowers, fruits, insects, kitchen items, trees, and types of weather. For half 
of the lists, the probe shared its category with one of the list items; categories were not otherwise repeated within a list (e.g., list: oak, rain, sock; probe: snow). Category lists ranged from one to seven items in length.

Procedure. For both tests, lists were presented verbally at a rate of one item per second. The probe followed the list after a 2-s pause. Participants responded "yes" or "no" according to whether a probe rhymed or was in the same category as a list item. The first two list lengths were preceded by three practice trials during which the experimenter gave feedback and highlighted any errors. For all patients, testing began with all of the one-item lists. If the patient was at least $75 \%$ accurate at this list length, testing continued with the two-item lists, with list length increasing in this manner until the patient's accuracy for a particular length fell below 75\%. Span was defined as the maximum length at which accuracy was $75 \%$; if this cutoff fell between two lengths, span was calculated with linear interpolation. The procedure for controls was the same except for two modifications designed to reduce the duration of the testing session. First, testing began with three-item lists for the category test and four-item lists for the rhyme test. Second, if participants correctly responded to 10 consecutive trials at any list length, they immediately proceeded to the next list length.

In a separate session, we used a single-item sorting task to examine whether the patients knew the category to which each test item belonged. We presented the patients with a display showing the category names alongside a photographed exemplar of each category. Each item from the main test was read out individually, and the patients pointed to the category to which they thought the item belonged. Control participants did not complete this section of the test.

Results. Performance on these tasks is given in Table 3. Both semantic STM patients performed within the normal range on the rhyme span task, and JB exceeded the mean span in controls. However, in the category test both patients failed on the three-item lists; this result indicated impaired semantic STM. Therefore, the category spans of these individuals were comparable with those of other reported semantic STM patients. They showed a dissociation between semantic and phonological retention, as expected. Both patients were largely accurate when they sorted individual items into categories, as they scored above $90 \%$. However, we rescored the category probe task, excluding any trials that featured a target or probe word the patient had not sorted correctly (see figures in parentheses in Table 3). This change had little effect on their accuracy, which suggested that their poor category spans were not simply a consequence of impaired category knowledge. ${ }^{2}$

Generally, the SA group performed poorly on the category and rhyme tasks; the 2 most severely impaired individuals were unable to respond accurately to the shortest lists. However, 3 patients (ME, PG, BB) showed a dissociation between the two tasks and scored more poorly on the category task. Low rhyme spans in this group of patients may reflect impairments to phonological storage. In line with their more severe semantic impairments, the 2 SA patients who showed the largest impairments were unable to sort the items into categories even when the items were presented individually. However, single-item sorting was relatively intact for the other patients. As was true for the semantic STM patients, exclusion of items that patients had not sorted correctly produced little improvement in the STM version of the task.

\section{ISR of Words and Nonwords}

Semantic STM patients show a reduction in the advantage for recall of words over nonwords. This reduction reflects an inability to use lexical-semantic information to aid STM (R. C. Martin \& Lesch, 1996).

Control participants. Control data for this experiment are reported in Jefferies, Jones, Bateman, and Lambon Ralph (2005). Ten participants between 50 and 70 years of age were tested. All had left full-time education when they were 14-16 years of age.

Materials and procedure. The test comprised lists of words and nonwords presented for ISR (taken from Gathercole, Pickering, Hall, \& Peaker, 2001). Controls and semantic STM patients received 20 word lists and 20 nonword lists in total. Half contained four items and half contained five items. Additionally, 5 SA patients were tested; as described above, these were the 5 individuals with most intact repetition skills. To avoid floor effects, we had them complete two-, three-, and four-item lists. There were 30 word lists and 30 nonword lists in total. The words and nonwords, which were not repeated in the course of the experiment, were blocked using an ABBA design and were presented auditorily at a rate of one item per second.

Results. Accuracy on this task is given in Figure 2. JB was highly accurate: He performed within the range of control participants on the word lists and actually exceeded the maximum control score when recalling nonwords ( $\mathrm{JB}=57 \%$; control range $=18 \%-46 \%$ ). The size of his lexicality effect (i.e., the difference between word and nonword accuracy) was significantly smaller than was that of controls, modified $t(9)=2.76, p<.05$. $\mathrm{ABU}$ recalled words more poorly than did controls, but his nonword recall was within the control range; he recalled $32 \%$ of nonwords accurately. ABU's lexicality effect was smaller than that of controls, modified $t(9)=3.64, p<.01$. The 5 patients with SA also showed a reduced advantage for word recall over nonword recall: There was a significant Lexicality $\times$ Group interaction when the SA patients were compared with controls, $F(1,13)=$ $16.8, p=.001$.

\section{ISR of Phonologically Similar and Dissimilar Lists}

An advantage for phonologically dissimilar material over similar-sounding items has often been taken as a marker of phonological coding in verbal STM (Conrad, 1964; Conrad \& Hull, 1964). Patients with phonological storage deficits present with reduced, or sometimes reversed, phonological similarity effects (R. C. Martin \& Breedin, 1992). However, normal effects of phonological similarity have been reported in semantic STM patients (R. C. Martin \& Lesch, 1996; R. C. Martin et al., 1994).

Control participants. Control data from 12 participants are reported in Jefferies et al. (2005). Ten of these participants also completed the previous experiment.

Materials and procedure. Participants recalled lists of phonologically similar and dissimilar letters in serial order. The phonologically similar set contained the letters $E, C, T, P, V, B, G$, and

\footnotetext{
2 The small improvement in JB's accuracy on the three-item lists was sufficient to exceed the $75 \%$ cutoff for this list length. However, when we tested JB with four-item lists he achieved a score of $61 \%$, which was considerably less accurate than the score of control participants.
} 
Table 3

Performance on Rhyme and Category Probe Tests

\begin{tabular}{|c|c|c|c|c|c|c|c|c|c|c|c|c|c|}
\hline \multirow[b]{2}{*}{ Task } & \multicolumn{2}{|c|}{ Semantic STM } & \multicolumn{9}{|c|}{ SA } & \multicolumn{2}{|c|}{ Controls $^{\mathrm{a}}$} \\
\hline & $\mathrm{JB}$ & $\mathrm{ABU}$ & $M^{\mathrm{a}}$ & JD & NY & $\mathrm{SC}$ & PG & BB & ME & LS & KA & $M$ & $S D$ \\
\hline Category span & $2.7^{*}$ & $2.7^{*}$ & 1.6 & $1.5^{*}$ & $2.6^{*}$ & $1.7^{*}$ & $1.6^{*}$ & $1.0^{*}$ & $1.3^{*}$ & $<1$ & $<1$ & 6.15 & 0.78 \\
\hline Rhyme span & 8.0 & 6.0 & 3.0 & $2.0^{*}$ & $2.4^{*}$ & $1.0^{*}$ & $3.0^{*}$ & 4.3 & 5.4 & $<1$ & $<1$ & 6.98 & 1.54 \\
\hline Single item sorting (\%) & 94 & 94 & 90 & 83 & 84 & 94 & 92 & 85 & 92 & 67 & 47 & & \\
\hline \multicolumn{14}{|l|}{ Category } \\
\hline Length 1 & $90(94)$ & $95(100)$ & & $83(81)$ & $85(89)$ & $85(84)$ & $100(100)$ & $75(72)$ & $80(88)$ & $60(67)$ & $45(25)$ & & \\
\hline Length 2 & $85(89)$ & $85(84)$ & & $70(80)$ & $85(88)$ & $70(70)$ & $60(60)$ & $60(61)$ & $60(71)$ & & & & \\
\hline Length 3 & $71(77)$ & $71(67)$ & & & $67(70)$ & & & & & & & & \\
\hline \multicolumn{14}{|l|}{ Rhyme } \\
\hline Length 1 & 100 & 100 & & 75 & 80 & 75 & 95 & 100 & 95 & 55 & 60 & & \\
\hline Length 2 & 95 & 85 & & 75 & 80 & & 75 & 95 & 75 & & & & \\
\hline Length 3 & 100 & 92 & & 42 & 67 & & 75 & 92 & 79 & & & & \\
\hline Length 4 & 92 & 75 & & & & & 71 & 88 & $58^{\mathrm{b}}$ & & & & \\
\hline Length 5 & 90 & 80 & & & & & & 50 & 85 & & & & \\
\hline Length 6 & 81 & 75 & & & & & & & 67 & & & & \\
\hline Length 7 & 75 & 61 & & & & & & & & & & & \\
\hline Length 8 & 75 & & & & & & & & & & & & \\
\hline
\end{tabular}

Note. Patient breakdown shows percentage accuracy at each stage of the test, and figures in parentheses exclude trials in which the target or probe was not sorted correctly. STM = short-term memory; $\mathrm{SA}=$ semantic aphasia; Controls = control participants. $\quad{ }^{*}$ Denotes impaired span (modified $t$ test, $p<$ $.05)$.

${ }^{\mathrm{a}}$ Excludes LS and KA. $\quad{ }^{\mathrm{b}}$ Testing was continued, as this score seemed to be anomalous.

$D$, and the dissimilar set consisted of the letters $W, S, Q, Y, R, J$, $F$, and $L$, following Knott et al. (2000). There were 40 lists; half contained four items and half contained six items. The phonologically similar and dissimilar letters were blocked using an ABBA design, and items were read aloud at a rate of one item per second for spoken recall.

Results. Mean item accuracy across both list lengths is shown in Figure 3. JB performed at a similar level to control participants, although ABU was rather less accurate. One possible explanation for ABU's difficulty on this task is that a small set of letters was repeated several times within the experiment, and this repetition led to high levels of proactive interference. This possibility would be consistent with a cognitive control explanation of ABU's STM problem (Hamilton \& Martin, 2005). In any case, it is important to note that both semantic STM patients showed a significant effect of phonological similarity: JB, $\chi^{2}(1, N=200)=8.72, p<.005$; ABU, $\chi^{2}(1, N=200)=22.3, p<.0001$ (all tests two-tailed). These results were in line with findings from previous semantic STM cases. Of the $5 \mathrm{SA}$ patients, 4 showed a significant effect of phonological similarity, $\chi^{2}(1, N=200)>5.01, p<.05$; the exception was LS, $\chi^{2}(1, N=200)=.02$. LS made a number of perseverative and letter completion errors (e.g., "BBC") that may have prevented the appearance of any effect.

\section{Discussion of Verbal STM Results}

In this section, we confirmed that the problems $\mathrm{JB}$ and $\mathrm{ABU}$ had with verbal STM were a result of impaired ability to use semantic information in recall. Their performance on tasks tapping phonological retention was good: Both $\mathrm{JB}$ and $\mathrm{ABU}$ demonstrated normal ISR of nonwords and were sensitive to phonological similarity in letter lists. JB's phonological retention capacity appeared completely spared, whereas ABU's scores were a little lower but not abnormal. In ISR, they showed a reduced lexicality effect; neither could recall word lists as well as their performance on nonword lists would predict. They were also impaired on the category probe test but performed well with the rhyming probes. This pattern of performance is consistent with that of other individuals with impaired semantic STM (R. C. Martin \& He, 2004; R. C. Martin et al., 1994). Indeed, the striking dissociation between phonological and semantic span in patient JB indicates a particularly pure case of the deficit.

Our patients were largely accurate at categorizing individual words, and their failure on the category STM task remained even when miscategorized items were excluded from the analysis. This result suggests that damage to core semantic representations (of the kind observed in SD) cannot explain the patients' semantic STM impairment. Instead, poor STM could be a consequence of disruption to a buffer specialized for the retention of lexicalsemantic information (R. C. Martin et al., 1994). An alternative view is that impairment to more general semantic control processes could be responsible, if one assumes that the STM version of the task placed greater demands on these processes than did the single-item task.

SA patients, who have semantic control impairments on a range of tasks, showed a broadly similar STM profile to that of the semantic STM patients. They were also impaired on the category probe task, even when errors in category knowledge were taken into account. Rhyme probe performance was variable but tended to be more intact than did the category test. SA patients also showed reduced lexicality effects and normal phonological similarity effects. This pattern suggests that they, like the semantic STM 


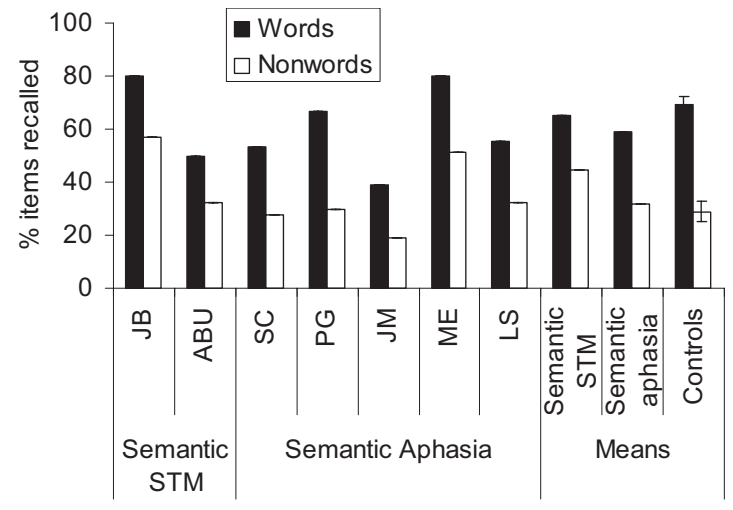

Figure 2. Individual and group data for word and nonword recall. JB, $\mathrm{ABU}$, and control participants received four- and five-item lists; other patients received two-, three-, and four-item lists. Bars indicate standard error for control participants. STM $=$ short-term memory.

patients, relied mainly on phonology and not semantics to complete ISR tasks.

\section{Section 2: Semantic Processing}

In this section of the study, we examined our semantic STM patients using a number of semantic memory assessments that did not load heavily on STM in order to determine whether their semantic problem was specific to STM or was a more general deficit. On each task, the semantic STM patients were compared with members of the SA case series to establish whether similar impairments were present in this group. There were three components to this section: Standard semantic battery. Patients received a battery of verbal and nonverbal semantic tasks that we have used previously with SA and SD patients (Bozeat et al., 2000; Jefferies \& Lambon Ralph, 2006). As have other individuals with semantic STM, JB and ABU scored well on these tests, and this led us to administer some more demanding assessments.

Speeded semantic tasks. Patients performed naming and synonym judgments at speed. Accuracy and reaction times revealed mild semantic impairments on these tasks.

Manipulation of control demands. Patients completed three tests considered to have high semantic control demands. This task explored whether their semantic deficit was consistent with an underlying impairment of semantic control.

\section{Standard Semantic Battery}

Method. Semantic processing was assessed with a 64-item semantic battery that assessed knowledge of the same 64 items across different input and output modalities and types of semantic judgment. The tests included the following: Camel and Cactus Test (Bozeat et al., 2000). The Camel and Cactus Test is a measure of semantic association similar to the Pyramids and Palm Trees Test (Howard \& Patterson, 1992); participants decide which of four semantically related items is most associated with a stimulus (e.g., does camel go with cactus, tree, sunflower, or rose). There were two versions: In one, the probe and choices were colored pictures; in the other, they were presented as written words that were read aloud by the examiner.
Spoken word-picture matching. Participants matched spoken names to pictures. There were nine semantically related foils. The target and foils were black-and-white line drawings from the Snodgrass and Vanderwart (1980) set.

Spoken picture naming. The patients were asked to name each item presented as a Snodgrass picture.

The Environmental Sounds Test (Bozeat et al., 2000). This test contains recorded sounds from six categories: domestic/foreign animals, human sounds, household items, vehicles, and musical instruments $(N=48)$. There were three conditions: matching sounds to pictures, matching sounds to written words, and matching spoken words to pictures. On each trial, the target was presented with nine within-category distractors.

$B N T$. Picture naming was assessed with the BNT. Patients were presented with all 60 pictures from the test; for any items they could not name, they were given a phonemic cue (typically, the first two phonemes of the word) and another opportunity to name. Although picture naming is not a purely semantic task, most researchers agree that semantic processing is a necessary first step in lexical production and that naming errors can be informative about the nature of a patient's deficit (Lambon Ralph, Moriarty, \& Sage, 2002; Schwartz, Dell, Martin, Gahl, \& Sobel, 2006). An error analysis was therefore conducted, in which data were combined from both naming tests. Single-word errors were classified as semantic if they shared a coordinate, superordinate, or associative relationship with the target and as phonological if they shared at least one phoneme with the target in the correct position in the word. Errors meeting both criteria were classed as mixed. An "other" category contained visual errors, perseverations, and other errors unrelated to the target. Errors of omission were counted separately, and we included circumlocutions in this category.

Results and discussion. Results are shown in Table 4. The semantic STM patients were largely intact on this battery; they showed mild impairments on the BNT and one other semantic test. In contrast, the SA patients showed a range of impairments, and all patients failed both verbal and nonverbal tests. The performance of the SA group on these tasks is described in more detail by Jefferies and Lambon Ralph (2006). In summary, patients showed consistency when the same task was presented in different modalities

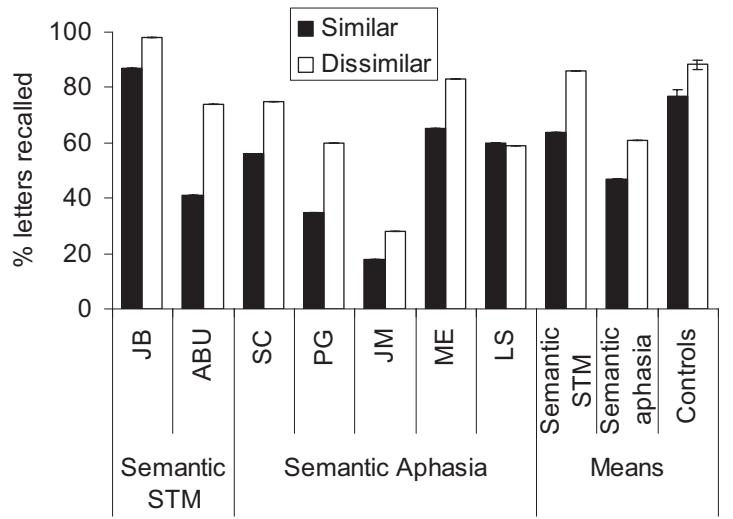

Figure 3. Individual and group data for phonological similarity experiment. Figure combines data from four- and six-item lists, except for patients PG and JM, who completed only four-item lists. Bars indicate standard error for control participants. STM $=$ short-term memory. 
Table 4

Performance on Standard Semantic Battery

\begin{tabular}{|c|c|c|c|c|c|c|c|c|c|c|c|c|c|c|c|c|}
\hline \multirow[b]{2}{*}{ Task } & \multirow[b]{2}{*}{ Max } & \multirow{2}{*}{$\begin{array}{l}\text { Normal } \\
\text { cutoff }\end{array}$} & \multicolumn{2}{|c|}{$\begin{array}{l}\text { Semantic } \\
\text { STM }\end{array}$} & \multicolumn{12}{|c|}{ SA } \\
\hline & & & $\mathrm{JB}$ & $\mathrm{ABU}$ & $M$ & $\mathrm{JD}$ & NY & $\mathrm{SC}$ & PG & $\mathrm{KH}$ & $\mathrm{BB}$ & $\mathrm{JM}$ & ME & MS & LS & KA \\
\hline \multicolumn{17}{|l|}{ 64-item battery } \\
\hline Naming & 64 & 59 & $58^{*}$ & 63 & 23.5 & $49^{*}$ & $54^{*}$ & $28^{*}$ & $46^{*}$ & $30^{*}$ & $10^{*}$ & $30^{*}$ & $5^{*}$ & $0^{*}$ & $5^{*}$ & $0^{*}$ \\
\hline Word-picture matching & 64 & 62 & 64 & $61^{*}$ & 51.0 & 64 & $60^{*}$ & $59^{*}$ & $58^{*}$ & $54^{*}$ & $54^{*}$ & $53^{*}$ & $50^{*}$ & $46^{*}$ & $37^{*}$ & $26^{*}$ \\
\hline Picture CCT & 64 & 52 & 59 & 57 & 36.1 & $38^{*}$ & $36^{*}$ & $46^{*}$ & $44^{*}$ & $46^{*}$ & $38^{*}$ & $37^{*}$ & $13^{*}$ & $37^{*}$ & $16^{*}$ & $46^{*}$ \\
\hline Word CCT & 64 & 56 & 62 & 57 & 37.2 & $38^{*}$ & $39^{*}$ & $56^{*}$ & $40^{*}$ & $41^{*}$ & $30^{*}$ & $37^{*}$ & $34^{*}$ & $42^{*}$ & $16^{*}$ & $36^{*}$ \\
\hline \multicolumn{17}{|l|}{ Environmental Sounds } \\
\hline Sound-picture & 48 & 36 & 41 & 42 & 27.8 & $23^{*}$ & $28^{*}$ & $32^{*}$ & $33^{*}$ & $30^{*}$ & $26^{*}$ & $24^{*}$ & $33^{*}$ & $28^{*}$ & $27^{*}$ & $22^{*}$ \\
\hline Sound-word & 48 & 33 & 44 & 44 & 25.2 & $26^{*}$ & 34 & $32^{*}$ & $25^{*}$ & $26^{*}$ & $27^{*}$ & $16^{*}$ & 35 & $25^{*}$ & $17^{*}$ & $14 *$ \\
\hline Word-picture & 48 & 46 & 48 & $46^{*}$ & 39.2 & $46^{*}$ & $44^{*}$ & $41^{*}$ & 47 & $44^{*}$ & $33^{*}$ & $43^{*}$ & $40^{*}$ & $37^{*}$ & $35^{*}$ & $21^{*}$ \\
\hline \multicolumn{17}{|l|}{$\mathrm{BNT}^{\mathrm{a}}$} \\
\hline Without cuing & 60 & $46^{\mathrm{b}}$ & $43^{*}$ & $43^{*}$ & & $38^{*}$ & $24^{*}$ & $11^{*}$ & $38^{*}$ & $20^{*}$ & $1^{*}$ & $19^{*}$ & $2^{*}$ & NT & $3^{*}$ & NT \\
\hline $\begin{array}{l}\text { Additional items } \\
\text { named with cue }\end{array}$ & - & - & $9 / 17$ & $15 / 17$ & & $8 / 22$ & $19 / 36$ & $10 / 49$ & $12 / 22$ & $6 / 40$ & $25 / 59$ & $13 / 41$ & $14 / 58$ & NT & $25 / 57$ & NT \\
\hline
\end{tabular}

Note. $\quad$ STM $=$ short-term memory; SA $=$ semantic aphasia; CCT $=$ Camels and Cactus Test (Bozeat et al., 2000); BNT $=$ Boston Naming

Test. $\quad$ *Denotes impaired scores (below 5 th percentile for BNT; $<2 S D$ below normal mean for all other tests).

${ }^{a}$ Kaplan et al. (1983). ${ }^{\mathrm{b}}$ Fifth percentile in adults age 55-65 (Zec, Burkett, Markwell, \& Larsen, 2007).

(e.g., sound-picture vs. sound-word matching) but were highly inconsistent when compared on tasks that required different types of semantic judgment (e.g., simple matching tasks vs. semantic association tasks). Performance on the Camel and Cactus association test was worst on trials on which it was difficult to elucidate the relevant semantic dimension or to reject the distractors. This result suggests that patients had difficulty selecting and attending to relevant semantic information. The high scores of our semantic STM patients prevented us from conducting similar analyses of their results.

The analysis of picture naming errors is presented in Table 5. Although JB and ABU made fewer errors overall, the error distribution was similar to that in the SA group. JB and ABU tended to make semantic or omission errors, although $\mathrm{ABU}$ also made a handful of phonological errors. Likewise, semantic errors and omissions accounted for the majority of errors in the SA group (the only major exception to this pattern was patient LS, who made frequent perseverative errors). This distribution of error types has been associated previously with semantic impairment in SD (Lambon Ralph, McClelland, Patterson, Galton, \& Hodges, 2001) and stroke aphasia (Dell, Schwartz, Martin, Saffran, \& Gagnon, 1997; Lambon Ralph et al., 2002; Schwartz et al., 2006).

\section{Speeded Picture Naming and Synonym Judgment}

The standard semantic battery provided little evidence for general semantic impairments in $\mathrm{JB}$ and $\mathrm{ABU}$, although their errors in picture naming were consistent with a semantic impairment. However, it was possible that mild semantic problems would be evident on more demanding tasks. As discussed in the Introduction, semantic STM patients $\mathrm{AB}$ and ML showed abnormalities in category judgments when they performed under timed conditions (R. C. Martin \& He, 2004; R. C. Martin et al., 1994). Here, we assessed semantic processing in JB and ABU using two timed semantic tasks: picture naming and synonym judgment. We chose these tests as they have different processing and response requirements (picture vs. verbal stimuli and verbal response vs. forced-choice selection). Impaired performance on both tasks would suggest a central semantic impairment rather than difficulties with a particular type of stimulus or mode of responding.

We also minimized the STM demands of the tasks. In the speeded category judgment task that revealed impairment in previous patients, the first item appeared visually for $1 \mathrm{~s}$ and the

Table 5

Errors in Picture Naming

\begin{tabular}{|c|c|c|c|c|c|c|c|c|c|c|c|c|}
\hline \multirow[b]{2}{*}{ Variable } & \multicolumn{2}{|c|}{ Semantic STM } & \multicolumn{10}{|c|}{ SA } \\
\hline & JB & $\mathrm{ABU}$ & $M$ & JD & NY & $\mathrm{SC}$ & PG & $\mathrm{KH}$ & $\mathrm{BB}$ & $\mathrm{JM}$ & ME & LS \\
\hline$\%$ items correct & 82 & 85 & 36 & 73 & 64 & 31 & 65 & 40 & 10 & 37 & 5 & 6 \\
\hline \multicolumn{13}{|c|}{ Error types as $\%$ of all errors } \\
\hline Semantic & 45 & 22 & 27 & 56 & 29 & 17 & 52 & 23 & 13 & 23 & 9 & 21 \\
\hline Omission & 45 & 61 & 50 & 26 & 53 & 51 & 23 & 68 & 79 & 50 & 85 & 17 \\
\hline Phonological & 0 & 11 & 5 & 0 & 4 & 15 & 9 & 0 & 2 & 12 & 1 & 3 \\
\hline Mixed & 5 & 0 & 5 & 3 & 4 & 9 & 7 & 7 & 3 & 8 & 2 & 0 \\
\hline Other & 5 & 6 & 13 & 15 & 9 & 7 & 9 & 3 & 3 & 8 & 3 & 59 \\
\hline
\end{tabular}

Note. $\mathrm{STM}=$ short-term memory; $\mathrm{SA}=$ semantic aphasia. 
second item was then presented verbally. This presentation raised the possibility that poor retention of the first item, rather than a central processing deficit, caused the decrement in performance. In our tests, items were presented visually via a computer monitor and remained on screen until a response was made.

Participants. Because accuracy and RT data were collected in this experiment, it was not possible to compare the semantic STM cases with the full set of SA patients, many of whom would not produce enough correct responses for an RT analysis. Therefore, the 4 patients with the mildest semantic impairments were selected to take part (JD, NY, SC, PG).

Method. Both tests were administered with a 15-in. laptop computer monitor running Eprime software. Participants were instructed to respond as quickly and accurately as possible, but no time limit was placed on responses. Picture naming involved 48 line drawings from the Snodgrass and Vanderwart (1980) set (24 living items and 24 nonliving). Presentation rate was controlled by the participant: When the spacebar was pressed, a picture appeared, accompanied by a 200-ms tone. For control participants and the semantic STM patients, time between the appearance of the picture and the onset of their first response was measured with an electronic voice key. The voice key was not used with the patients with SA, who often made extraneous responses and comments before naming; instead, a digital recording of the session was analyzed with WavePad software (NCH Swiftsound, Canberra, Australia) to obtain response latencies.

For synonym judgment (Jefferies, Patterson, Jones, \& Lambon Ralph, 2008), participants saw a stimulus word and three possible target words and indicated which target word was synonymous with the stimulus. The stimulus word was presented near the top of the screen, and the three possible responses appeared simultaneously beneath it. There were 96 trials in total. Participants responded by pressing 1, 2, or 3 on the computer keyboard. They controlled the presentation rate by pressing the spacebar when they were ready for each trial, and the computer recorded latencies from the onset of the stimulus until a response was made. To ensure that patients were able to read the test items accurately, we presented the stimuli as above in a separate session and asked patients to read them aloud as quickly as possible.

Results. Accuracy and reaction times are shown in Table 6. In picture naming, JB correctly named fewer pictures than did control participants, as did the SA patients. ABU's accuracy did not differ from that of the controls. The reaction times for incorrect re- sponses were discarded, as were voice key errors (one response each for JB and $\mathrm{ABU}$ and $1.9 \%$ of responses for controls). Outliers beyond two standard deviations of a participant's mean were excluded. The analysis revealed that both semantic STM patients were significantly slower to name picture than were controls. The slowing was particularly evident in JB. In addition to being less accurate, 3 patients with SA were abnormally slow.

Reading of the synonym judgment stimuli was largely accurate: JB read $97 \%$ of the words correctly and ABU read $91 \%$ of them correctly. In the SA group, JD, NY, and PG were $99 \%, 82 \%$, and $91 \%$ accurate respectively, but patient SC was unable to read most of the words and therefore could not complete the synonym judgment task. All of the patients were significantly impaired when judging synonyms. The accuracy of JB and ABU was within the range of the patients with mild SA. Impairment on the synonym judgment task did not appear to be related to the patients' mild deficits in reading the items aloud, as all of the patients remained significantly impaired when trials in which the target or probe word were read incorrectly were excluded from the analysis. To analyze RT, we removed outliers that deviated by more than two standard deviations from each participant's mean. JB responded more slowly than did controls, but ABU was in the normal range. The patients with SA were very slow to complete this task.

Discussion. These timed tasks revealed deficits of semantic processing in JB and ABU. JB named pictures less accurately than did controls, and both patients were impaired on the synonym judgment task. In addition, reaction times were abnormally slow for both patients when they named pictures and for JB when he made synonym judgments. The magnitude of these impairments approached that of the milder aphasic cases. These findings suggest that semantic processing is not entirely normal in patients with semantic STM deficits, even when they perform tasks with a minimal STM load. The presence of deficits on two rather different tasks suggests that the patients' problems are not an artifact of a particular method of testing but represent a general problem in accessing semantic information.

The final three experiments in this study were intended to provide further evidence for this semantic impairment and to investigate the nature of the deficit. Specifically, we were interested in whether the semantic deficit in JB and ABU reflected a problem with semantic control, as we have hypothesized for individuals with multimodal semantic impairments following stroke,

Table 6

Accuracy and Reaction Times in Speeded Semantic Tasks

\begin{tabular}{|c|c|c|c|c|c|c|c|c|}
\hline \multirow[b]{2}{*}{ Variable } & \multicolumn{2}{|c|}{ Semantic STM } & \multicolumn{4}{|c|}{ SA } & \multicolumn{2}{|c|}{ Controls } \\
\hline & JB & $\mathrm{ABU}$ & JD & NY & SC & PG & $M$ & $S D$ \\
\hline \multicolumn{9}{|l|}{ Picture naming } \\
\hline Accuracy $(\%)$ & $71^{*}$ & 90 & $58^{*}$ & $58^{*}$ & $46^{*}$ & $73^{*}$ & 93 & 3.7 \\
\hline $\mathrm{RT}(\mathrm{ms})$ & $5,149^{*}$ & $1,712^{*}$ & 1,222 & $2,692^{*}$ & $9,695^{*}$ & $2,142^{*}$ & 1,038 & 215 \\
\hline \multicolumn{9}{|c|}{ Synonym judgment ${ }^{\mathrm{a}}$} \\
\hline Accuracy $(\%)$ & $81^{*}$ & $71^{*}$ & $68^{*}$ & $67^{*}$ & $\mathrm{NC}$ & $85^{*}$ & 98 & 2.0 \\
\hline $\mathrm{RT}(\mathrm{ms})$ & $3,802^{*}$ & 2,053 & $12,592^{*}$ & $8,383^{*}$ & $\mathrm{NC}$ & $9,103^{*}$ & 1,731 & 288 \\
\hline
\end{tabular}

Note. $\quad$ STM $=$ short-term memory; $\mathrm{SA}=$ semantic aphasia; Controls $=$ control participants; NC $=$ test not completed. $*$ Denotes impaired performance (modified $t$ test, $p<.05$ ).

a Jefferies et al. (2008). 
or was more consistent with degraded semantic knowledge, as is the case for patients with SD (Rogers et al., 2004). Because our semantic STM patients seemed to have very mild semantic deficits, we focused on semantically driven word production tasks, as these tend to be more sensitive to semantic impairment than are receptive tests.

\section{Phonologically Cued Picture Naming}

Providing phonological cues often improves picture naming in aphasia. This facilitation can be explained in a number of ways, depending on the characteristics of the patients in question. One effect of providing phonological cues is to boost activation of the target word above that of its competitors (Lambon Ralph, Sage, \& Roberts, 2000; Myers Pease \& Goodglass, 1978; Patterson, Purrell, \& Morton, 1983); this may be particularly helpful for individuals with semantic control deficits that affect selection of semantic information. The SA patients in this study made numerous associative errors in picture naming (e.g., squirrel $\rightarrow$ "nuts"). This result suggests that pictures did elicit some relevant semantic activation but that the patients failed to engage control processes in order to regulate this activation and select the appropriate response. Accordingly, they showed substantial phonological cuing effects in naming, which suggests that they often could resolve competition between potential responses with the aid of additional external constraints (Jefferies, Patterson, \& Lambon Ralph, 2008). Furthermore, providing miscues that primed a semantic competitor had a negative effect on performance (Noonan, Jefferies, Corbett, Hopper, \& Lambon Ralph, 2008). Patients with SD, on the other hand, showed little benefit from cuing, as their degraded semantic systems provided insufficient initial semantic activation for cues to aid selection. JB and ABU did show effects of phonological cues in our background semantic testing. However, the patients were fairly accurate and cues were given only when they made an incorrect initial response, so there were relatively few trials in which cues were presented.

In this experiment we examined phonemic cuing in a more systematic manner and provided cues before a response was made. In addition, we examined the effect of miscues that were inconsistent with the target response. These miscues might be expected to exacerbate control problems by boosting the activation of competing representations. We were not able to test the SA group in this experiment; however, several members of the case series were tested with a similar experiment by Noonan et al. (2008) and showed significant cuing effects and negative effects of miscues.

Method. Patients were presented with the 60 pictures of the BNT (Kaplan et al., 1983) on three separate occasions. On each occasion, one third of the items were preceded by a correct phonemic cue, one third by a phoneme that cued a semantic competitor (e.g., picture of a tree with $/ b /$ from bush), and one third by no cue. Correct and incorrect cues were interspersed, so that patients were unaware of which cues were helpful. Over the three sessions, each picture was paired once with a correct cue, once with a miscue, and once with no cue.

Results. JB named 40/60 pictures without cues, 46/60 with correct cues, and 35/60 when miscued. ABU named $41 / 60$ pictures when no cue was provided, 51/60 with a correct cue, and 37/60 when miscued. One-tailed McNemar tests revealed that both patients named more pictures when provided with correct cues than with miscues $(p<.001)$. ABU showed a significant benefit of correct cues compared with no cue $(p<.001)$; this difference just failed to reach statistical significance in JB $(p=.073)$. JB made a total of 19 semantic errors in the experiment, and his remaining 40 errors consisted of omissions or circumlocutions. ABU made 29 semantic errors and 17 omission/circumlocution errors but also made 4 phonological errors and 1 apparent visual error (artist's palette $\rightarrow$ "heart"). Semantic errors made by both patients tended to be consistent with the presented cue (e.g., picture of octopus and $/ s / \rightarrow$ "squid").

Discussion. These effects are consistent with an impaired control explanation of the patients' deficits. Initial phonemic information is likely to boost the activation of representations consistent with the cue. When a correct cue was presented, this increased the likelihood of the patients retrieving the correct name, in line with the idea that their mild naming impairment resulted from competition between highly active competitors. Conversely, miscues led to fewer correct responses and a tendency to produce semantic errors consistent with the cue, which suggests that miscues promoted activation of semantic competitors that the patients failed to resolve. In another study, patients with SA responded in a similar manner to phonemic cues and miscues (Noonan et al., 2008). This response pattern provided further evidence for similarities between the SA patients and semantic STM patients. The behavior of JB and $\mathrm{ABU}$ is less consistent with degradation to the semantic knowledge store itself, as SD patients with this impairment are insensitive to phonological cuing (Funnell \& Hodges, 1996; Hodges et al., 1992; Jefferies, Patterson, et al., 2008).

\section{Verbal Fluency}

In verbal fluency tasks, participants produce as many exemplars of a given taxonomic category or of words beginning with a certain letter as possible within $60 \mathrm{~s}$. The open-ended nature of the task means that responses are weakly constrained by the stimulus and emphasizes the need for internal cognitive control over word retrieval (Baldo \& Shimamura, 1998; Chertkow \& Bub, 1990; Funnell \& Hodges, 1996). The fact that individuals must have the cognitive control required to retrieve a number of words on the basis of a single cue and must inhibit previous responses so that they are not repeated implicates a role for selection mechanisms (Hirshorn \& Thompson-Schill, 2006). Impaired performance on verbal fluency tasks would therefore be consistent with a deficit to semantic control.

Participants. As above, KA and MS were excluded from this test due to their inability to produce verbal output. Patient performance was compared with control data from 30 healthy participants between 54 and 82 years of age.

Method. Patients were given the name of a category or a letter of the alphabet and were asked to produce as many exemplars as possible within $1 \mathrm{~min}$, without repetition. There were eight categories (animals, birds, fruit, breeds of dog, household items, tools, vehicles, and types of boat) and three letters $(F, A, S)$.

Results and discussion. Figure 4 shows the number of correct responses by each patient. JB and ABU produced significantly fewer exemplars than did control participants in category fluency, and this result provided further evidence for a mild semantic deficit, modified $t$ test for each patient, $t(29)=2.77, p=.01$. ABU was also impaired at the letter fluency task, $t(29)=2.45, p<.05$, 


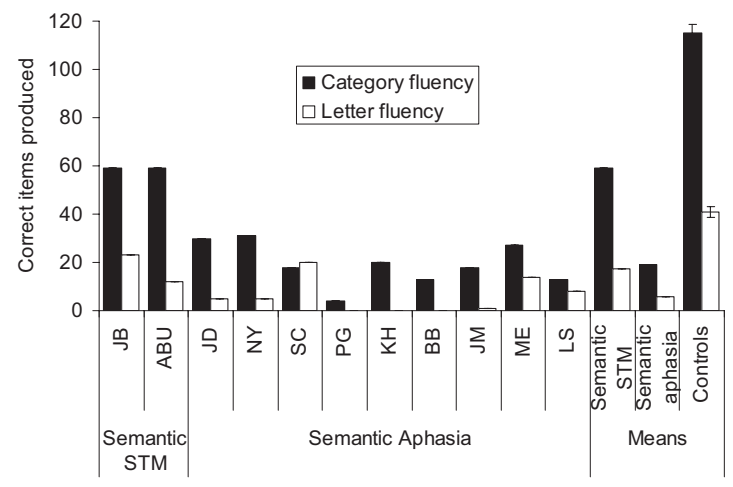

Figure 4. Verbal fluency. Bars indicate $1 S E$ for controls. STM = short-term memory.

and all of the SA patients were severely impaired on both tasks, $t(29)>2.28, p<.05$. The only exception was SC's score on letter fluency, which was not significantly impaired.

Although verbal production deficits may have contributed to the SA patients' difficulties on these tasks, this is a less convincing explanation of the poor performance of JB and $\mathrm{ABU}$, because they both displayed fluent spontaneous speech in conversation. It is more difficult to entirely exclude the possibility that STM deficits contributed to the patients' reduced fluency. Neither patient showed a tendency to repeat responses within a trial, which suggested they retained information about their previous responses. Moreover, both patients produced appropriate exemplars over the entire 60-s period, and this fact indicated that they retained the category/letter throughout. However, STM problems might still have contributed to their reduced production rate if, for example, the category was lost midtrial and had to be reconstructed on the basis of memory for previous responses.

An alternative explanation is that the poor performance of the STM patients stemmed from their difficulties in using control processes to selectively retrieve relevant lexical and semantic information. Damage to semantic representations themselves, as seen in SD, also impairs category fluency (Hodges et al., 1992; Marczinski \& Kertesz, 2006). However, semantic deterioration in SD is graded by frequency; patients tend not to produce lowfrequency exemplars in category fluency or other tasks. In contrast, both semantic STM patients produced infrequent words among their responses (e.g., ABU produced "elk" for animals and JB produced "ptarmigan" for birds).

\section{Verb Generation}

In the verb generation task, participants are presented with a noun and must generate a verb that is semantically associated with it. Although in some cases this task is no more difficult than simple word association (e.g., chair $\rightarrow$ "sit"), there are many common nouns for which there are no obvious associated verbs (e.g., ant $\rightarrow$ ?). By manipulating the number of associates and the strength of association between nouns and verbs, researchers have used this task to investigate the effect of competing responses (Thompson-Schill et al., 1997, 1998) and the need to engage in controlled semantic retrieval when cue association strength is low (R. C. Martin \& Cheng, 2006). Trials in which there are no strongly associated verbs and those in which there are multiple strongly associated verbs are assumed to depend on semantic control processes to a greater extent than are trials that feature a single strongly associated verb.

Here, we administered R. C. Martin and Cheng's (2006) version of the task, which independently varied number and strength of verb associates. We had two aims in this experiment. First, as the verb generation task is a demanding test of semantic access, we expected to obtain further evidence for semantic impairment in JB and $\mathrm{ABU}$. Second, the pattern of performance across conditions should provide clues to the nature of the semantic impairment, specifically whether it related to selection among competitors or to retrieval based on weakly specified cues.

Participants. In addition to JB and $\mathrm{ABU}$, we tested the four least affected individuals from the SA group (JD, NY, SC, PG).

Materials. A noun was presented and participants attempted to produce a verb that was semantically associated. The nouns were those used by R. C. Martin and Cheng (2006), and they differed along two variables: selection demand (the degree of competition between likely responses) and association strength (the strength of the relationship between the noun and most probable verb response). In the low selection condition, each noun was highly associated with a single verb (e.g., apple $\rightarrow$ "eat"). In the high selection-high association condition, each noun was strongly associated with two different verbs (e.g., door $\rightarrow$ "open," door $\rightarrow$ "close"). Finally, the high selection-low association condition contained nouns for which there were no strongly associated verbs: Each noun was weakly associated with a number of verbs (e.g., rug $\rightarrow$ "lay," rug $\rightarrow$ "roll"). There were 15 nouns in each condition, which were presented in a random order.

Procedure. Words were presented on a 15-in. laptop computer monitor. The noun appeared visually in the center of the screen along with simultaneous auditory presentation. The word remained on screen until a response was made or the participants indicated that they were unable to think of a suitable word. Eight practice trials preceded the main test. If patients made a number of nonverb responses, they were reminded of the instruction to respond only with verbs. Responses were digitally recorded and were analyzed later to obtain reaction times.

Results. Only patients JB and ABU could complete this task successfully. None of the patients with SA were able to comply with the task instructions; they either did not respond at all or consistently gave multiword or nonverb responses, despite prompting and repeated explanation. One patient (PG) began by giving very slow but valid responses but found the experience of generating these words so effortful that he requested that the test be abandoned halfway through. Results for JB and ABU are given in Table 7, alongside data from the control group. Although reaction time is reported for the patients, it is based on small numbers of correct responses and we therefore focus our analysis on accuracy data.

JB performed flawlessly in the low selection condition, but he was slightly less accurate for high selection-high association nouns and substantially error prone for the high selection-low association nouns. His accuracy in this condition was significantly worse than in the low selection condition, $\chi^{2}(1, N=30)=9.31$, $p<.005$. ABU was less accurate than JB in all three conditions, but he also performed most poorly in the high selection-low association condition, which differed significantly from the low 
Table 7

Verb Generation Task

\begin{tabular}{|c|c|c|c|c|}
\hline \multirow[b]{2}{*}{ Condition } & \multirow[b]{2}{*}{ JB } & \multirow[b]{2}{*}{$\mathrm{ABU}$} & \multicolumn{2}{|c|}{ Controls } \\
\hline & & & $M$ & $S D$ \\
\hline \multicolumn{5}{|c|}{ Low selection (e.g., apple $\rightarrow$ "eat") } \\
\hline Accuracy & $15 / 15$ & $11 / 15$ & 13.8 & 1.87 \\
\hline Reaction time (ms) & 2,359 & 4,089 & 1,510 & 332 \\
\hline \multicolumn{5}{|c|}{$\begin{array}{l}\text { High selection-high association (e.g., } \\
\text { door } \rightarrow \text { "open," door } \rightarrow \\
\text { "close") }\end{array}$} \\
\hline Accuracy & $13 / 15$ & $10 / 15$ & 13.9 & 2.28 \\
\hline Reaction time (ms) & 2,911 & 4,141 & 1,376 & 258 \\
\hline \multicolumn{5}{|c|}{$\begin{array}{l}\text { High selection-low association (e.g., } \\
\text { rug } \rightarrow \text { "lay," rug } \rightarrow \text { "roll") }\end{array}$} \\
\hline Accuracy & $8 / 15$ & $4 / 15^{*}$ & 13.4 & 2.95 \\
\hline Reaction time (ms) & 3,660 & 6,786 & 2,042 & 708 \\
\hline
\end{tabular}

Note. Controls $=$ control participants. ${ }^{*}$ Denotes impaired accuracy (modified $t$ test, $p<.05$ ).

selection condition, $\chi^{2}(1, N=30)=8.57, p<.005$. The majority of errors by JB were failures to respond, though he did give a nonverb response to one item (roof $\rightarrow$ "tiles"). ABU tended to make nonverb responses ( $81 \%$ of all errors), which were usually associated nouns (e.g., ear $\rightarrow$ "lobe").

Discussion. This task provided further evidence for mild semantic impairment in patient $\mathrm{ABU}$, who was impaired relative to control participants when generating verbs on the basis of a weakly associated noun. Although JB was not impaired at a statistically significant level, he showed a similar pattern and performed least accurately in the weakly associated condition. The STM impairment could conceivably have contributed to the patients' poor performance on this task, because although the stimuli remained on screen throughout each trial, the task instructions had to be retained throughout the test session. However, this possibility does not straightforwardly account for the differences in performance across conditions. Our patients showed particularly poor performance when they generated verbs in the low-association condition, and the same pattern was shown by semantic STM patient ML (R. C. Martin \& Cheng, 2006). This result suggests that semantic STM cases have difficulty retrieving semantic information based on weakly associated cues, as is consistent with the hypothesis that poor semantic control underpins their problems. Indeed, 4 SA patients with more severely impaired semantic control were unable to complete the task.

\section{General Discussion}

This study investigated verbal STM and semantic processing in 2 patients with semantic STM deficits. The semantic STM patients resembled previously reported cases in terms of their STM profile (R. C. Martin \& He, 2004; R. C. Martin et al., 1994): Both performed normally on a rhyme probe STM task but were impaired when they responded to category probes. They showed a reduced advantage for words over nonwords in ISR but exhibited normal effects of phonological similarity. The major finding of the study was that both patients showed mild deficits on semantic tasks that did not appear to be a direct consequence of their STM impairment. In common with previous semantic STM patients, ours were unimpaired on standard untimed semantic tasks. However, under time constraints, deficiencies in accuracy and reaction time were apparent. Demanding verbal fluency and verbgeneration tasks also revealed impairments.

The semantic STM patients were compared with a case series of SA patients, all of whom had more severe semantic impairments associated with poor control over semantic representations (Jefferies \& Lambon Ralph, 2006). There was some evidence for commonalities between this group and the semantic STM patients. The SA patients showed a similar pattern of STM impairment, in that they showed reduced lexicality effects and performed poorly on the category probe task. Deficits on the category probe task did not seem to be a consequence of impoverished semantic knowledge: Patients performed poorly on trials that contained targets and probes they could correctly sort into categories. There was evidence of more widespread semantic deficits in these patients and, like the semantic STM patients, they were very impaired on verbal fluency tasks that required a high degree of cognitive control. The verb-generation task, another demanding task that involves selection and controlled retrieval, proved too challenging even for those patients who were least affected by SA.

Our interpretation of the present findings is that STM deficits in the two semantic STM cases we have described are a consequence of more general deficiencies in semantic processing. Specifically, they might arise from a mild impairment to control processes involved in the access and manipulation of semantic information. On this view, both semantic STM patients and SA patients with more severe semantic problems fall on a continuum of impairment in which the same aspects of controlled semantic processing are damaged but to different extents. Mild impairments to semantic control have a detrimental effect on a the ability to deal with the semantic aspects of verbal STM tasks (and other semantic tasks that place large demands on controlled processing) but leave performance on less cognitively challenging semantic tasks intact. More severe control deficits affect less demanding single-word semantic tasks (e.g., picture naming, word-picture matching) in addition to control over semantic STM. In this section, we deal with alternative interpretations of the data from JB and $\mathrm{ABU}$ and then consider precisely how control might play a role in semantic STM tasks.

An obvious challenge to the explanation we have put forward is that our patients' deficits are adequately explained as impaired retention of lexical-semantic information over time. We attempted to minimize retention demands by presenting stimuli visually throughout each trial. Nevertheless, some subtle STM demands may have remained. For example, in the fluency tasks patients must self-generate responses over $60 \mathrm{~s}$, and doing so requires them to remember the task instructions throughout. In the synonym judgment task, the probe and three choices were available in visual form during each trial, but one might argue that an intact lexicalsemantic buffer is necessary for the individual to attend to each pair of words long enough to compare them. The retention and control/selection demands of semantic tasks are difficult to separate, because most complex tasks involve the maintenance of task instructions over time or the ability to attend to multiple stimuli simultaneously. It is in part these requirements that make semantic tasks demanding of cognitive control. More work is needed to separate which aspects of these patients' deficits can be attributed to control processes and which to maintenance of task-relevant 
representations over time. Nevertheless, there is some unambiguous evidence from a test without STM demands: Picture naming, a simple task involving single stimuli, was mildly impaired in both patients.

Another possibility is that JB and ABU have experienced mild damage to the semantic store itself. Our primary reason for rejecting this hypothesis is that the patients' STM deficits did not correspond to their knowledge of individual word meanings. In the category probe task, both patients demonstrated good knowledge of individual word meanings, and removing the few words they misclassified from the analysis did not alter the outcome of the STM test. This finding contrasts with that for SD patients and individuals with nonprogressive impairment of semantic knowledge, which suggests that impaired knowledge of word meanings accounts for the deficit in STM (Caza, Belleville, \& Gilbert, 2002; Forde \& Humphreys, 2002; Jefferies et al., 2004).

If the core deficit in STM cases is one of impaired cognitive control, why does this deficit have a disproportionate effect on STM tasks? STM tasks might place particular demands on control processes because of the number of representations that must be maintained simultaneously. Individuals must resist interference from items presented on previous trials, and there is evidence that semantic STM patients can have particular difficulty with this aspect of STM (Hamilton \& Martin, 2005, 2007; R. C. Martin \& Lesch, 1996). The category probe task has additional elements that emphasize controlled processing. It requires patients to retain a number of word meanings but also to compare each of those meanings with the probe and ascertain whether a match is present. There are at least two ways in which this comparison might be accomplished. Individuals might access semantic representations of list items and compare the features of each list item with the features of the probe. Sufficient feature overlap would indicate a category match. However, decisions must be made on the basis of the categories defined within the task and not merely by gross similarity (e.g., lilac and maple are rather similar but are not a match because one is classed as a flower and the other as a tree). So, alternatively, individuals might use the semantic information retrieved to determine a category for each word and compare these categories with the category derived from the probe.

Either method involves competition and selection demands of the kind envisaged by Thompson-Schill et al. $(1997 ; 1998)$, as a number of features or categories are retained simultaneously. Competition between features from different items could disrupt the assignment of categories or reduce the activation of individual features, which would cause matches to be missed. Additionally, because only nine categories are used in the task and individual items are repeated, the possibility of proactive interference from previous trials is large. More work is needed to determine precisely which aspects of the task are disrupted in semantic STM patientsindeed, it is possible that different processes are deficient in different patients-but it seems plausible that semantic control may play in significant role in these deficits.

One obvious question is why the category probe task was disrupted in semantic STM patients but the rhyme probe task, which has apparently similar control requirements, was not. One possibility is that the control mechanisms recruited to deal with semantic representations are distinct from those involved in phonological judgments (Devlin, Matthews, \& Rushworth, 2003; Fiez, 1997). Another is that the phonological version of the task was not as demanding as its semantic counterpart. Healthy controls performed slightly better on the rhyming task, which suggests that this task is less taxing. The detection of two words that rhyme might be easier because the same criterion can be applied in every case: Rhyming words always share identical phonology in the same place in both words. Detecting two concepts that belong to the same category is a more complex judgment involving the retrieval and comparison of a number of features or their categories. The criterion to detect a match varies considerably according to the particular items being considered (e.g., goat, sheep, and elephant all belong to the animal category but goats and elephants are much less similar to one another than are goats and sheep). Category judgments might therefore place greater demands on controlled processing than on rhyme detection, which would explain why the two tasks dissociated in our patients.

In support of the idea that semantic and phonological representations share control processes, semantic STM patient ML was affected by both semantic and phonological similarity in probed recall and showed abnormal interference effects for letters, which lack semantic content (Hamilton \& Martin, 2007). There was also some evidence that cognitive control was impaired more generally in $\mathrm{JB}$ and $\mathrm{ABU}$. Both patients showed weakness on tests of nonverbal executive function.

How might semantic control deficits be realized in implemented models of semantic and lexical processing? A detailed consideration of this issue is beyond the scope of this investigation, but we can offer some suggestions as to how control problems might map onto existing models. One model that has been used to account for picture naming and repetition in aphasia is the interactive activation framework of Dell and colleagues (Foygel \& Dell, 2000; Schwartz et al., 2006). In the model, activation spreads between semantic, lexical, and phonological nodes through bidirectional connections. Naming begins with activation of a set of semantic feature nodes corresponding to a particular object. Activation spreads through the network for a given number of time units, after which a jolt of activation is given to the lexical node with the highest activation level; this boosts its activation significantly, so that it far outstrips that of its competitors. Activation then flows once again through network; after further time steps, jolts are applied to the most active phonological nodes, which are taken as the phonemes of the response. A lesion that affected the initial activation jolt could potentially mimic some of the effects seen in $\mathrm{SA}^{3}$

Reducing the activation boost applied to the most active lexical node would increase competition between the target word and other lexical representations, which accumulate some activation as a result of their semantic similarity to the target. Alternatively, noise could be added to the jolt process, so that competing lexical representations sometimes receive an activation boost rather than the target. In either case, the result would be a reduction in accuracy and increase in semantic errors. This kind of damage would be alleviated by cuing, as is seen in the patients. Activating the initial phonemic node of the target at the same time as its semantic features would increase the activation of the target but not its semantically related competitors and thus would combat the effects of the disrupted jolt process.

\footnotetext{
${ }^{3}$ We are grateful to an anonymous reviewer for this suggestion.
} 
Although activation jolts are a specific feature of the interactive activation model, they illustrate a general principle that could be incorporated into models of semantic processing to account for control processes. Essentially, the jolts are the result of an external source of regulation over activation in the language system, which we attribute to semantic control. To account for performance across a range of tasks, this regulation must be applied flexibly, with different features or representations being activated or inhibited depending on the task being performed. ${ }^{4}$

There remains one major difference between the semantic STM patients reported here and existing patients in the literature, and this is in the location of their lesions. Semantic STM problems have previously been associated with lesions to left prefrontal cortex. Patient ML, for example, had a large frontoparietal lesion that included the left inferior frontal gyrus (Hamilton \& Martin, 2007), an area strongly associated with semantic control in neuroimaging studies (e.g., Badre, Poldrack, Pare-Blagoev, Insler, \& Wagner, 2005; Devlin et al., 2003; Moss et al., 2005; ThompsonSchill et al., 1997; Wagner et al., 2001). Our patient JB had damage to the posterior temporal lobe, and ABU's damage, although harder to interpret, also appeared to spare the frontal lobe. One might conclude on the basis of this neuroanatomical evidence that semantic representations rather than control processes were affected.

Why then do we favor the semantic control explanation? First, because the mismatch between the patients' comprehension of words and ability to hold them in STM makes damage to semantic representations an unlikely explanation of the patients' deficits. Second, there is growing evidence that cognitive control is supported by a distributed network of brain regions and does not rely exclusively on prefrontal cortex. Peers et al. (2005), for example, studied visual STM in patients with frontal and parietal lesions. Patients with parietal lesions displayed slower visual processing speeds than did patients with frontal lesions. However, attentional control-defined as the ability to ignore irrelevant stimuli-was not selectively impaired in the group with frontal lesions; instead, it correlated with overall lesion size in both groups. Peers et al. postulated that frontal and parietal cortices play similar roles in top-down attentional control. Our own studies of semantic control have revealed similar findings in the semantic domain; patients with temporoparietal lesions showed deficits in semantic control similar to those shown by patients with damage in the prefrontal cortex (Jefferies et al., 2007; Jefferies \& Lambon Ralph, 2006; Jefferies, Patterson, et al., 2008). Similarly, neuroimaging studies of cognitive and executive control have reported temporoparietal activations (Collette et al., 2005, 2006; Garavan et al., 2000). Activity in this region has also been observed in controlled semantic tasks, for example, when resolving semantic ambiguity in sentences (Rodd, Davis, \& Johnsrude, 2005) and when selectively attending to particular aspects of a stimulus (Cristescu, Devlin, \& Nobre, 2006). Although the role of posterior regions in control over semantic memory specifically requires further study, it seems that an absence of frontal damage need not rule out problems with control of semantic representations.

As a final point, we would not wish to claim that all STM deficits can be explained through poor control mechanisms. Deficits of phonological STM have been linked to faulty rehearsal processes and impairment of a phonological buffer (e.g., R. C. Martin et al., 1994; Vallar \& Baddeley, 1984; Warrington \&
Shallice, 1969). There is also considerable evidence that verbal STM problems in individuals with SD are closely related to the degradation of their semantic knowledge (e.g., Patterson et al., 1994). However, poor cognitive control might account for those cases in which semantic aspects of STM are impaired in the absence of either a phonological STM impairment or degraded semantic knowledge. We have demonstrated in two such patients that mild semantic processing deficits can be revealed with sufficiently sensitive and demanding tasks, and this finding raises the possibility that semantic STM deficits might reflect a problem with control over semantic representations.

\footnotetext{
${ }^{4}$ For an implementation of this sort of task-dependent regulation in a nonlanguage domain, see Rougier, Noelle, Braver, Cohen, and O'Reilly (2005)
}

\section{References}

Albert, M., Goodglass, H., Helms, N. A., Rubens, A. B., \& Alexander, M. N (1981). Clinical aspects of dysphasia. New York/Berlin: Springer-Verlag.

Baddeley, A. D., \& Hitch, G. J. (1974). Working memory. In G. Bower (Ed.), The psychology of learning and motivation (Vol. 8, pp. 47-90). New York: Academic Press.

Baddeley, A. D., Thompson, N., \& Buchanan, M. (1975). Word length and the structure of short term memory. Journal of Verbal Learning and Verbal Behaviour, 14, 575-589.

Badre, D., Poldrack, R. A., Pare-Blagoev, E. J., Insler, R. Z., \& Wagner, A. D. (2005). Dissociable controlled retrieval and generalized selection mechanisms in ventrolateral prefrontal cortex. Neuron, 47, 907-918.

Badre, D., \& Wagner, A. D. (2002). Semantic retrieval, mnemonic control, and prefrontal cortex. Behavioural and Cognitive Neuroscience Reviews, 1, 206-218

Baldo, J. V., \& Shimamura, A. P. (1998). Letter and category fluency in patients with frontal lobe lesions. Neuropsychology, 12, 259-267.

Berthier, M. L. (2000). Transcortical aphasias. Hove, England: Psychology Press.

Berthier, M. L. (2001). Unexpected brain-language relationships in aphasia: Evidence from transcortical sensory aphasia associated with frontal lobe lesions. Aphasiology, 15, 99-130.

Bourassa, D. C., \& Besner, D. (1994). Beyond the articulatory loop: A semantic contribution to serial order recall of subspan lists. Psychonomic Bulletin \& Review, 1, 122-125.

Bozeat, S., Lambon Ralph, M. A., Patterson, K., Garrard, P., \& Hodges, J. R. (2000). Non-verbal semantic impairment in semantic dementia. Neuropsychologia, 38, 1207-1215.

Bunge, S. A., Wendelken, C., Badre, D., \& Wagner, A. D. (2005). Analogical reasoning and prefrontal cortex: Evidence for separable retrieval and integration mechanisms. Cerebral Cortex, 15, 239-249.

Burgess, P., \& Shallice, T. (1996). Bizarre responses, rule detection and frontal lobe lesions. Cortex, 32, 241-259.

Caza, N., Belleville, S., \& Gilbert, B. (2002). How loss of meaning with preservation of phonological word form affects immediate serial recall performance: A linguistic account. Neurocase, 8, 255-273.

Chertkow, H., \& Bub, D. (1990). Semantic memory loss in dementia of Alzheimer's type: What do various measures measure? Brain, 113, 397417.

Chertkow, H., Bub, D., Deaudon, C., \& Whitehead, V. (1997). On the status of object concepts in aphasia. Brain and Language, 58, 203-232.

Collette, F., Hogge, M., Salmon, E., \& Van der Linden, M. (2006). Exploration of the neural substrates of executive functioning by neuroimaging. Neuroscience, 139, 209-221.

Collette, F., Olivier, L., Van der Linden, M., Laureys, S., Delfiore, G., 
Luxen, A., et al. (2005). Involvement of both prefrontal and inferior parietal cortex in dual task performance. Cognitive Brain Research, 24, 237-251.

Collette, F., \& Van der Linden, M. (2002). Brain imaging of the central executive component of working memory. Neuroscience and Biobehavioural Reviews, 26, 105-125.

Conrad, R. (1964). Acoustic confusion in immediate memory. British Journal of Psychology, 55, 75-84.

Conrad, R., \& Hull, A. J. (1964). Information, acoustic confusion and memory span. British Journal of Psychology, 55, 429-432.

Crawford, J. R., \& Garthwaite, P. H. (2002). Investigation of the single case in neuropsychology: Confidence limits on the abnormality of test scores and test score differences. Neuropsychologia, 40, 1196-1208.

Cristescu, T. C., Devlin, J. T., \& Nobre, A. C. (2006). Orienting attention to semantic categories. Neuroimage, 33, 1178-1187.

Crosson, B., Rao, S. M., Woodley, S. J., Rosen, A. C., Bobholz, J. A., Mayer, A., et al. (1999). Mapping of semantic, phonological, and orthographic verbal working memory in normal adults with functional magnetic resonance imaging. Neuropsychology, 13, 171-187.

Dell, G. S., Schwartz, M. F., Martin, N., Saffran, E. M., \& Gagnon, D. A. (1997). Lexical access in aphasic and nonaphasic speakers. Psychological Review, 104, 801-838.

De Renzi, E., Faglioni, P., Scotti, G., \& Spinnler, H. (1972). Impairment in associating colour to form, concomitant with aphasia. Brain, 95, 293-304.

Devlin, J. T., Matthews, P. M., \& Rushworth, M. F. S. (2003). Semantic processing in the left inferior prefrontal cortex: A combined functional magnetic resonance imaging and transcranial magnetic stimulation study. Journal of Cognitive Neuroscience, 15, 71-84.

Fiez, J. A. (1997). Phonology, semantics, and the role of the left inferior prefrontal cortex. Human Brain Mapping, 5, 79-83.

Forde, E. M. E., \& Humphreys, G. W. (2002). The role of semantic knowledge in short-term memory. Neurocase, 8, 13-27.

Foygel, D., \& Dell, G. S. (2000). Models of impaired lexical access in speech production. Journal of Memory and Language, 43, 182-216.

Freedman, M. L., \& Martin, R. C. (2001). Dissociable components of short-term memory and their relation to long-term learning. Cognitive Neuropsychology, 18, 193-226.

Funnell, E., \& Hodges, J. R. (1996). Deficits of semantic memory and executive control: Evidence for differing effects upon naming in dementia. Aphasiology, 10, 687-709.

Gainotti, G., Miceli, G., \& Caltagirone, C. (1979). The relationship between conceptual and semantic-lexical disorders in aphasia. International Journal of Neuroscience, 10, 45-50.

Gainotti, G., Silveri, M. C., Villa, G., \& Caltagirone, C. (1983). Drawing objects from memory in aphasia. Brain, 106, 613-622.

Garavan, H., Ross, T. J., Li, S. L., \& Stein, E. A. (2000). A parametric manipulation of central executive functioning. Cerebral Cortex, 10, 585592.

Gathercole, S. E., Pickering, S. J., Hall, M., \& Peaker, S. M. (2001). Dissociable lexical and phonological influences on serial recognition and serial recall. Quarterly Journal of Experimental Psychology, 54A, 1-30.

Goodglass, H. (1983). The assessment of aphasia and related disorders (2nd ed.). Philadelphia: Lea \& Febiger.

Hamilton, A. C., \& Martin, R. C. (2005). Dissociations among tasks involving inhibition: A single-case study. Cognitive Affective \& Behavioral Neuroscience, 5, 1-13.

Hamilton, A. C., \& Martin, R. C. (2007). Proactive interference in a semantic short-term memory deficit: Role of semantic and phonological relatedness. Cortex, 43, 112-123.

Hart, J., \& Gordon, B. (1990). Delineation of single-word semantic comprehension deficits in aphasia with anatomical correlation. Annals of Neurology, 27, 226-231.

Hazy, T. E., Frank, M. J., \& O'Reilly, R. C. (2006). Banishing the homunculus: Making working memory work. Neuroscience, 139, 105-118.
Hirshorn, E. A., \& Thompson-Schill, S. L. (2006). Role of the left inferior frontal gyrus in covert word retrieval: Neural correlates of switching during verbal fluency. Neuropsychologia, 44, 2547-2557.

Hodges, J. R., Graham, N., \& Patterson, K. (1995). Charting the progression in semantic dementia: Implications for the organization of semantic memory. Memory, 3, 463-495.

Hodges, J. R., Patterson, K., Oxbury, S., \& Funnell, E. (1992). Semantic dementia: Progressive fluent aphasia with temporal lobe atrophy. Brain, 115, 1783-1806.

Howard, D., \& Patterson, K. (1992). Pyramids and Palm Trees: A test of semantic access from pictures and words. Bury St Edmunds, England: Thames Valley Test Company.

Hulme, C., Maughan, S., \& Brown, G. D. A. (1991). Memory for familiar and unfamiliar words: Evidence for a long-term memory contribution to shortterm memory span. Journal of Memory and Language, 30, 685-701.

Hulme, C., Roodenrys, S., Schweickert, R., Brown, G. D. A., Martin, S., \& Stuart, G. (1997). Word-frequency effects on short-term memory tasks: Evidence for a redintegration process in immediate serial recall. Journal of Experimental Psychology: Learning, Memory, and Cognition, 23, $1217-1232$

Jefferies, E., Baker, S. S., Doran, M., \& Lambon Ralph, M. A. (2007). Refractory effects in stroke aphasia: A consequence of poor semantic control. Neuropsychologia, 45, 1065-1079.

Jefferies, E., Frankish, C., \& Lambon Ralph, M. A. (2006). Lexical and semantic binding in verbal short-term memory. Journal of Memory and Language, 54, 81-98.

Jefferies, E., Hoffman, P., Jones, R., \& Lambon Ralph, M. A. (2008). The impact of semantic impairment on verbal short-term memory in stroke aphasia and semantic dementia: A comparative study. Journal of Memory and Language, 58, 66-87.

Jefferies, E., Jones, R., Bateman, D., \& Lambon Ralph, M. A. (2004). When does word meaning affect immediate serial recall in semantic dementia? Cognitive, Affective and Behavioural Neuroscience, 4, 20-42.

Jefferies, E., Jones, R. W., Bateman, D., \& Lambon Ralph, M. A. (2005). A semantic contribution to nonword recall? Evidence for intact phonological processes in semantic dementia. Cognitive Neuropsychology, 22, $183-212$.

Jefferies, E., \& Lambon Ralph, M. A. (2006). Semantic impairment in stroke aphasia vs. semantic dementia: A case-series comparison. Brain, 129, 2132-2147.

Jefferies, E., Patterson, K., Jones, R. W., \& Lambon Ralph, M. A. (2008). Comprehension of concrete and abstract words in semantic dementia. Manuscript submitted for publication.

Jefferies, E., Patterson, K., \& Lambon Ralph, M. A. (2008). Deficits of knowledge versus executive control in semantic cognition: Insights from cued naming. Neuropsychologia, 46, 649-658.

Kaplan, E., Goodglass, H., \& Weintraub, S. (1983). Boston Naming Test. Philadelphia: Lea \& Febiger.

Knott, R., Patterson, K., \& Hodges, J. R. (1997). Lexical and semantic binding effects in short-term memory: Evidence from semantic dementia. Cognitive Neuropsychology, 14, 1165-1218.

Knott, R., Patterson, K., \& Hodges, J. R. (2000). The role of speech production in auditory-verbal short-term memory: Evidence from progressive fluent aphasia. Neuropsychologia, 38, 125-142.

Lambon Ralph, M. A., McClelland, J. L., Patterson, K., Galton, C. J., \& Hodges, J. R. (2001). No right to speak? The relationship between object naming and semantic impairment: Neuropsychological abstract evidence and a computational model. Journal of Cognitive Neuroscience, 13, $341-356$

Lambon Ralph, M. A., Moriarty, L., \& Sage, K. (2002). Anomia is simply a reflection of semantic and phonological impairments: Evidence from a case-series study. Aphasiology, 16, 56-82.

Lambon Ralph, M. A., Sage, K., \& Roberts, J. (2000). Classical anemia: A 
neuropsychological perspective on speech production. Neuropsychologia, 38, 186-202.

Majerus, S., Norris, D., \& Patterson, K. (2007). What does a patient with semantic dementia remember in verbal short-term memory? Order and sound but not words. Cognitive Neuropsychology, 24, 131-151.

Marczinski, C. A., \& Kertesz, A. (2006). Category and letter fluency in semantic dementia, primary progressive aphasia, and Alzheimer's disease. Brain and Language, 97, 258-265.

Martin, N., \& Ayala, J. (2004). Measurements of auditory-verbal STM span in aphasia: Effects of item, task, and lexical impairment. Brain and Language, 89, 464-483.

Martin, N., \& Saffran, E. (1990). Repetition and verbal STM in transcortical sensory aphasia: A case study. Brain and Language, 39, 254-288.

Martin, N., \& Saffran, E. M. (1997). Language and auditory-verbal shortterm memory impairments: Evidence for common underlying processes. Cognitive Neuropsychology, 14, 641-682.

Martin, R. C., \& Breedin, S. D. (1992). Dissociations between speech perception and phonological short-term memory deficits. Cognitive Neuropsychology, 9, 509-534.

Martin, R. C., \& Cheng, T. (2006). Selection demands versus association strength in the verb generation task. Psychonomic Bulletin \& Review, 13, 396-401.

Martin, R. C., \& He, T. (2004). Semantic short-term memory and its role in sentence processing: A replication. Brain and Language, 89, 76-82.

Martin, R. C., \& Lesch, M. (1996). Associations and dissociations between language impairment and list recall: Implications for models of STM. In S. E. Gathercole (Ed.), Models of short-term memory (pp. 149-178). East Sussex, England: Psychology Press.

Martin, R. C., Lesch, M. F., \& Bartha, M. C. (1999). Independence of input and output phonology in word processing and short-term memory. Journal of Memory and Language, 41, 3-29.

Martin, R. C., Shelton, J., \& Yaffee, L. S. (1994). Language processing and working memory: Neuropsychological evidence for separate phonological and semantic capacities. Journal of Memory and Language, 33, 83-111.

McCarthy, R. A., \& Warrington, E. K. (1987). The double dissociation of short-term memory for lists and sentences: Evidence from aphasia. Brain, 110, 1545-1563.

McCarthy, R. A., \& Warrington, E. K. (2001). Repeating without semantics: Surface dysphasia? Neurocase, 7, 77-87.

Milner, B. (1964). Effects of different brain lesions on card sorting: The role of the frontal lobes. Archives of Neurology, 9, 100-110.

Miyake, A., Friedman, N. P., Emerson, M. J., Witzki, A. H., Howerter, A., \& Wager, T. D. (2000). The unity and diversity of executive functions and their contributions to complex "frontal lobe" tasks: A latent variable analysis. Cognitive Psychology, 41, 49-100.

Moss, H. E., Abdallah, S., Fletcher, P., Bright, P., Pilgrim, L., Acres, K., et al. (2005). Selecting among competing alternatives: Selection and retrieval in the left inferior frontal gyrus. Cerebral Cortex, 15, 1723-1735.

Mummery, C. J., Patterson, K., Price, C. J., Ashburner, J., Frackowiak, R. S. J., \& Hodges, J. R. (2000). A voxel-based morphometry study of semantic dementia: Relationship between temporal lobe atrophy and semantic memory. Annals of Neurology, 47, 36-45.

Myers Pease, D., \& Goodglass, H. (1978). The effects of cueing on picture naming in aphasia. Cortex, 14, 178-189.

Nestor, P. J., Fryer, T. D., \& Hodges, J. R. (2006). Declarative memory impairments in Alzheimer's disease and semantic dementia. Neuroimage, 30, 1010-1020.

Noonan, K. A., Jefferies, E., Corbett, F., Hopper, S., \& Lambon Ralph, M. A. (2008). Elucidating the nature of deregulated semantic cognition in semantic aphasia: Evidence for the roles of the prefrontal and temporoparietal cortices. Manuscript submitted for publication.

O'Reilly, R. C., Braver, T. S., \& Cohen, J. D. (1999). A biologically based computational model of working memory. In A. Miyake \& S. Shah (Eds.),
Models of working memory: Mechanisms of active maintenance and executive control (pp. 375-411). New York: Cambridge University Press.

O'Reilly, R. C., \& Soto, R. (2002). A model of the phonological loop: Generalization and binding. In T. G. Dietterich, S. Becker, \& Z. Ghahramani (Eds.), Advances in Neural Information Processing Systems (NIPS) 14 (pp. 83-90). Cambridge, MA: MIT Press.

Patterson, K., Graham, N., \& Hodges, J. R. (1994). The impact of semantic memory loss on phonological representations. Journal of Cognitive Neuroscience, 6, 57-69.

Patterson, K., Nestor, P. J., \& Rogers, T. T. (2007). Where do you know what you know? The representation of semantic knowledge in the human brain. Nature Reviews Neuroscience, 8, 976-987.

Patterson, K., Purrell, C., \& Morton, J. (1983). Facilitation of word retrieval in aphasia. In C. Code \& D. Muller (Eds.), Aphasia therapy. London: Edward Arnold.

Peers, P. V., Ludwig, C. J. H., Rorden, C., Cusack, R., Bonfiglioli, C. Bundesen, C., et al. (2005). Attentional functions of parietal and frontal cortex. Cerebral Cortex, 15, 1469-1484.

Poirier, M., \& Saint-Aubin, J. (1995). Memory for related and unrelated words: Further evidence on the influence of semantic factors in immediate serial recall. Quarterly Journal of Experimental Psychology, 48A, $384-404$.

Raven, J. C. (1962). Coloured progressive matrices sets $A, A B$, $B$. London: H. K. Lewis.

Raven, J. C. (1992). Standard progressive matrices: 1992 edition. Windsor, England: NFER-Nelson.

Rey, A. (1941). L'examen psychologique dans le cas d'encaphalopathie traumatique [Psychological examination in traumatic encephalopathy] Arch Psychologie, 28, 286-340.

Robertson, I. H., Ward, T., Ridgeway, V., \& Nimmo-Smith, I. (1994). The Test of Everyday Attention. Flempton, England: Thames Valley Test Company.

Rodd, J. M., Davis, M. H., \& Johnsrude, I. S. (2005). The neural mechanisms of speech comprehension: FMRI studies of semantic ambiguity. Cerebral Cortex, 15, 1261-1269.

Rogers, T. T., Lambon Ralph, M. A., Garrard, P., Bozeat, S., McClelland, J. L., Hodges, J. R., et al. (2004). Structure and deterioration of semantic memory: A neuropsychological and computational investigation. Psychological Review, 111, 205-235.

Rougier, N. P., Noelle, D. C., Braver, T. S., Cohen, J. D., \& O'Reilly, R. C. (2005). Prefrontal cortex and flexible cognitive control: Rules without symbols. Proceedings of the National Academy of Sciences, USA, 102, $7338-7343$.

Saffran, E. M. (2000). The organization of semantic memory: In support of a distributed model. Brain and Language, 71, 204-212.

Saygin, A. P., Dick, F., Wilson, S. M., Dronkers, N., \& Bates, E. (2003). Neural resources for processing language and environmental sounds. Brain, 126, 928-945.

Schwartz, M. F., Dell, G. S., Martin, N., Gahl, S., \& Sobel, P. (2006). A case-series test of the interactive two-step model of lexical access: Evidence from picture naming. Journal of Memory and Language, 54, 228-264.

Snodgrass, J. G., \& Vanderwart, M. (1980). A standardized set of 260 pictures: Norms for name agreement, image agreement, familiarity and visual complexity. Journal of Experimental Psychology: Human Learning and Memory, 6, 174-215.

Snowden, J. S., Goulding, P. J., \& Neary, D. (1989). Semantic dementia: A form of circumscribed cerebral atrophy. Behavioural Neurology, 2, 167-182.

Spinnler, H., \& Vignolo, L. A. (1966). Impaired recognition of meaningful sounds in aphasia. Cortex, 2, 337-348.

Stuss, D. T., Levine, B., Alexander, M. P., Hong, J., Palumbo, C., Hamer, L., et al. (2000). Wisconsin Card Sorting Test performance in patients with focal frontal and posterior brain damage: Effects of lesion location 
and test structure on separable cognitive processes. Neuropsychologia, 34, 388-402.

Thompson-Schill, S. L., Bedny, M., \& Goldberg, R. F. (2005). The frontal lobes and the regulation of mental activity. Current Opinion in Neurobiology, 15, 219-224.

Thompson-Schill, S. L., D'Esposito, M., Aguirre, G. K., \& Farah, M. J. (1997). Role of left inferior prefrontal cortex in retrieval of semantic knowledge: A re-evaluation. Proceedings of the National Academy of Sciences, USA, 94, 14792-14797.

Thompson-Schill, S. L., D'Esposito, M., \& Kan, I. P. (1999). Effects of repetition and competition on activity in left prefrontal cortex during word generation. Neuron, 23, 513-522.

Thompson-Schill, S. L., Swick, D., Farah, M. J., D’Esposito, M., Kan, I. P., \& Knight, R. T. (1998). Verb generation in patients with focal frontal lesions: A neuropsychological test of neuroimaging findings. Proceedings of the National Academy of Sciences, USA, 95, 15855-15860.

Vallar, G., \& Baddeley, A. D. (1984). Phonological short-term store, phonological processing and sentence comprehension: A neuropsychological case-study. Cognitive Neuropsychology, 1, 121-141.

Wagner, A. D., Pare-Blagoev, E. J., Clark, J., \& Poldrack, R. A. (2001). Recovering meaning: Left prefrontal cortex guides controlled semantic retrieval. Neuron, 31, 329-338.

Walker, I., \& Hulme, C. (1999). Concrete words are easier to recall than abstract words: Evidence for a semantic contribution to short-term serial recall. Journal of Experimental Psychology: Learning, Memory, and Cognition, 25, 1256-1271.
Warrington, E. K., \& Cipolotti, L. (1996). Word comprehension: The distinction between refractory and storage impairments. Brain, 119, 611-625.

Warrington, E. K., \& James, M. (1991). The Visual Object and Space Perception battery. Bury St. Edmunds, England: Thames Valley Test Company.

Warrington, E. K., \& McCarthy, R. A. (1983). Category-specific access dysphasia. Brain, 106, 859-878.

Warrington, E. K., \& Shallice, T. (1969). Selective impairment of auditory verbal short-term memory. Brain, 92, 885-896.

Warrington, E. K., \& Shallice, T. (1979). Semantic access dyslexia. Brain, $102,43-63$.

Wechsler, D. (1987). Wechsler Memory Scale-Revised (WMS-R). New York: Psychological Corporation.

Woollams, A., Cooper-Pye, E., Hodges, J. R., \& Patterson, K. (2008). Anomia: A doubly typical signature of semantic dementia. Neuropsychologia, 46, 2503-2514.

Zec, R. F., Burkett, N. R., Markwell, S. J., \& Larsen, D. L. (2007). Normative data stratified for age, education, and gender on the Boston Naming Test. Clinical Neuropsychologist, 21, 617-637.

Received October 4, 2007

Revision received July 22, 2008

Accepted August 10, 2008

\section{Correction to Szpunar et al. (2008)}

In the article "Testing During Study Insulates Against the Buildup of Proactive Interference," by Karl K. Szpunar, Kathleen B. McDermott, and Henry L. Roediger III (Journal of Experimental Psychology: Learning, Memory, and Cognition, Vol. 34, pp. 1392-1399), incorrect figures were printed due to an error in the production process. A corrected version of Figure 5 is presented below. To see the complete article with the correct figures, please go to http://dx.doi.org/10.1037/a0013082

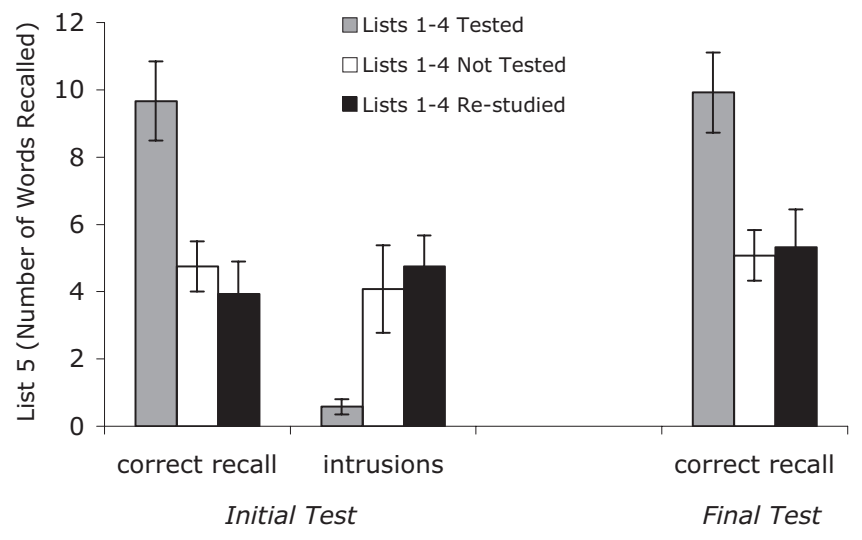

DOI: $10.1037 / \mathrm{a} 0014896$ 